\title{
On the Coefficients of the Singularities of the Solution of Maxwell's Equations near Polyhedral Edges
}

\author{
Boniface Nkemzi \\ Department of Mathematics, University of Buea, Buea, Cameroon \\ Correspondence should be addressed to Boniface Nkemzi; nkemzi@yahoo.com
}

Received 26 August 2015; Accepted 17 December 2015

Academic Editor: Haipeng Peng

Copyright ( 2016 Boniface Nkemzi. This is an open access article distributed under the Creative Commons Attribution License, which permits unrestricted use, distribution, and reproduction in any medium, provided the original work is properly cited.

\begin{abstract}
The solution fields of Maxwell's equations are known to exhibit singularities near corners, crack tips, edges, and so forth of the physical domain. The structures of the singular fields are well known up to some undetermined coefficients. In twodimensional domains with corners and cracks, the unknown coefficients are real constants. However, in three-dimensional domains the unknown coefficients are functions defined along the corresponding edges. This paper proposes explicit formulas for the computation of these coefficients in the case of two-dimensional domains with corners and three-dimensional domains with straight edges. The coefficients of the singular fields along straight edges of three-dimensional domains are represented in terms of Fourier series. The formulas presented are aimed at the numerical approximation of the coefficients of the singular fields. They can also be used for the construction of adaptive $H^{1}$-nodal finite-element procedures for the efficient numerical treatment of Maxwell's equations in nonsmooth domains.
\end{abstract}

\section{Introduction}

Unlike the regularity analysis for elliptic boundary value problems in domains with geometric singularities, where there exists a unified theory based on the shift theorem (see [1-3]), the regularity analysis of the solution of Maxwell's equations has several interpretations. Most papers are concerned with the $H^{1}$-regularity for nonconvex domains, and it is shown that the main singularity is the gradient of singular functions associated with the Laplace equation; see, for example, Birman and Solomyak [4], Bonnet-Ben Dhia et al. [5], Moussaoui [6], Hazard and Lohrengel [7], Hazard and Lenoir [8], and Lohrengel [9]. Costabel in [10] went further to address the $H^{1 / 2}$-regularity for Lipschitz domains.

The $H^{2}$-regularity of the solution of Maxwell's equations has been considered, for example, by Nkemzi [11-14]. To be more precise, the asymptotic behaviour of the solution near axisymmetric edges and their efficient numerical treatment by means of the Fourier-finite-element method on graded meshes were analyzed in [11, 14]. In [12] the problem was considered in axisymmetric domains with conical points and the asymptotic behaviour of the solution near the conical points was analyzed. Here explicit representation formulas for the coefficients of the singularities were derived. In [13] Maxwell's equations in polygonal domains were considered and formulas for the coefficients of the singularities were derived. The present paper considers Maxwell's equations in three-dimensional domains with polyhedral edges and the main focus is on the explicit description of the coefficients of the singularities. Unlike in two-dimensional case and the case of conical points where the space of the singular solutions is finite dimensional and the coefficients of the singularities are some real numbers, in three-dimensional domains with edges the space of the singular solutions is infinite dimensional and the coefficients of the singularities are functions defined along the edges. Here the space of the singular solutions along polyhedral edges is completely described.

It should be noted that a more rigorous regularity analysis based on the shift theorem for the Maxwell equations in plane domains with corners and in polyhedral domains has been 
carried out by Costabel et al. in $[15,16]$. They showed, using the classical Mellin analysis, a technique due to Kondratev [17], that, for a given current density in $H^{s-1}, s \geq 1$, the electromagnetic fields either belong to the space $H^{s+1}$ or else can be split into a regular part in $H^{s+1}$ and an explicitly defined singular part up to some unknown coefficients. They represented the singular fields along the edge in a tensor product form. However, it has been shown (see, e.g., Heinrich [18]) that the numerical treatment of boundary value problems in three-dimensional domains with edges is more efficient if the singular solutions along the edges are expressed in nontensor product forms.

Asymptotic analysis of solutions of boundary value problems near geometric singularities is usually carried out by considering only the principal part of the differential operator with frozen coefficients; see, for example, $[16,17]$. This is due to the fact that the qualitative behaviour of the solutions near geometric singularities depends only on the principal part of the operator, the geometry of the domain near the singularity, and the nature of the boundary conditions. On the other hand, it is known that the coefficients in the asymptotic expansion are linear continuous functions of the right hand side datum of the differential equation; see $[2,3]$. Thus, formulas for the coefficients are derived by taking into account the exact differential operator under consideration. These coefficients, especially the leading coefficients, determine the actual strength of the singularities and are the principal indicators for material damage analysis, for example, fracture analysis. Hence, their computation is not only important for the numerical treatment of the boundary value problem but also important for practical applications.

In this paper we extend the $H^{2}$-regularity analysis of the solution of time-harmonic Maxwell equations in plane domains with corners (see [13]) to three-dimensional domains with polyhedral edges. The main concern is to derive explicit computable formulas for the coefficients of the singularities. The singular functions along polyhedral edges are expressed in Fourier series and in nontensor product forms which are more suitable for constructing accurate finiteelement solutions. The results presented here can be used to construct postprocessing procedures for the $H^{1}$-nodal finiteelement treatment of Maxwell's equations in domains with geometric singularities and with optimal accuracy.

This paper is organised as follows. In Section 2 we introduce the boundary value problem and related function spaces and address the issue of existence and uniqueness of the weak solution. In Section 3 we formulate in Theorems 5 and 6 the main results concerning the regularity properties of Maxwell's equations in two-dimensional domains with corners and three-dimensional domains with polyhedral edges, respectively. Sections 4 and 5 contain detailed proofs of Theorems 5 and 6, respectively.

\section{The Boundary Value Problem and Functional Tools}

We consider as model problem the electromagnetic fields $\{\mathbf{E}, \mathbf{B}\}$ (E the electric field and $\mathbf{B}$ the magnetic field) of timeharmonic Maxwell's equations in a simply connected and bounded domain $\Omega \subset \mathbb{R}^{3}$ with Lipschitz boundary $\Gamma$ containing an isotropic and homogeneous medium subject to perfect conductor boundary conditions $[5,19,20]$ :

$$
\begin{aligned}
-i \epsilon \mu \omega \mathbf{E}+\operatorname{curl} \mathbf{B} & =\mu \mathbf{J}+\mu \sigma \mathbf{E} \quad \text { in } \Omega, \\
i \omega \mathbf{B}+\operatorname{curl} \mathbf{E} & =\mathbf{0} \quad \text { in } \Omega, \\
\mathbf{E} \wedge \mathbf{n} & =\mathbf{0}, \\
\mathbf{B} \cdot \mathbf{n} & =0,
\end{aligned}
$$

on $\Gamma$,

where the domain related parameters $\epsilon>0, \mu>0$, and $\sigma>$ 0 are, respectively, the dielectric permittivity, magnetic permeability, and electric conductivity, $\mathbf{J}$ is a given divergencefree current density, that is, $\operatorname{div} \mathbf{J}=0, \omega \neq 0$ is the pulsation of the electromagnetic fields, $i$ is the imaginary unity, and $\mathbf{n}$ denotes the unit outward normal on the boundary $\Gamma$.

If we suppose temporarily that the vector function $\mathbf{J}$ is sufficiently smooth, then system (1) can be written as two decoupled systems in terms of the magnetic field $\mathbf{B}$ and the electric field $\mathbf{E}$ as follows:

$$
\begin{aligned}
\operatorname{curl} \operatorname{curl} \mathbf{B}-\alpha^{2} \mathbf{B}=\mathbf{h} & \text { in } \Omega, \\
\mathbf{B} \cdot \mathbf{n}=0 & \text { on } \Gamma, \\
\text { curl curl } \mathbf{E}-\alpha^{2} \mathbf{E}=\mathbf{f} & \text { in } \Omega, \\
\mathbf{E} \wedge \mathbf{n}=\mathbf{0} & \text { on } \Gamma,
\end{aligned}
$$

where $\mathbf{h}=\mu \operatorname{curl} \mathbf{J}, \alpha^{2}=\omega^{2} \mu(\epsilon-i \sigma / \omega)$, and $\mathbf{f}=-i \omega \mu \mathbf{J}$.

It follows directly from (1), (2), and (3) that the solution of problem (2) can be derived from the solution of problem (3) and vice versa. Thus it suffices to solve or analyze only one of the problems. Subsequently we will dedicate our analysis to the boundary value problem (3). For the Hilbert space formulation of (3) we introduce the function spaces; see $[19,21]$ :

$$
\begin{aligned}
\mathscr{H}(\operatorname{curl}, \Omega) & :=\left\{\mathbf{v} \in\left(L_{2}(\Omega)\right)^{3}: \operatorname{curl} \mathbf{v} \in\left(L_{2}(\Omega)\right)^{3}\right\}, \\
\mathscr{H}_{0}(\operatorname{curl}, \Omega): & =\{\mathbf{v} \in \mathscr{H}(\operatorname{curl}, \Omega): \mathbf{v} \wedge \mathbf{n}=\mathbf{0} \text { on } \Gamma\},
\end{aligned}
$$

equipped with the norm

$$
\|\mathbf{v}\|_{\mathscr{H}(\operatorname{curl}, \Omega)}:=\left(\|\mathbf{v}\|_{\left(L_{2}(\Omega)\right)^{3}}^{2}+\|\operatorname{curl} \mathbf{v}\|_{\left(L_{2}(\Omega)\right)^{3}}^{2}\right)^{1 / 2}
$$

The variational formulation of problem (3) is as follows.

Find $\mathbf{u} \in \mathscr{H}_{0}(\operatorname{curl}, \Omega)$ such that

$$
\begin{aligned}
& a(\mathbf{u}, \mathbf{v}):=(\operatorname{curl} \mathbf{u}, \operatorname{curl} \mathbf{v})-\alpha^{2}(\mathbf{u}, \mathbf{v})=(\mathbf{f}, \mathbf{v})=: f(\mathbf{v}), \\
& \forall \mathbf{v} \in \mathscr{H}_{0}(\operatorname{curl}, \Omega),
\end{aligned}
$$


where $(\cdot, \cdot)$ denotes the usual scalar product in the Hilbert space $L_{2}(\Omega)$ of complex-valued functions. The well-posedness of problem (6) is addressed by the following theorem; see also $[19,22,23]$.

Theorem 1. Let $\Omega \subset \mathbb{R}^{3}$ be a bounded domain with at least Lipschitz continuous boundary $\Gamma$. Suppose $\alpha \neq 0$ and $\alpha^{2}$ is not an eigenvalue of the operator curl curl with electric boundary condition. Then, for any $\mathbf{f} \in\left(L_{2}(\Omega)\right)^{3}$, there exists a unique solution $\mathbf{u} \in \mathscr{H}_{0}(\mathrm{curl}, \Omega)$ of the variational problem (6). Moreover, the solution satisfies the estimate

$$
\|\mathbf{u}\|_{\left(L_{2}(\Omega)\right)^{3}}^{2}+\|\operatorname{curl} \mathbf{u}\|_{\left(L_{2}(\Omega)\right)^{3}}^{2} \leq C\|\mathbf{f}\|_{\left(L_{2}(\Omega)\right)^{3}}^{2} .
$$

We will always assume where necessary, without explicitly stating so, that the conditions of Theorem 1 are satisfied.

We observe that the operator $\mathbf{u} \mapsto$ curl curl $\mathbf{u}-\alpha^{2} \mathbf{u}$ from (2) and (3) is not elliptic. However, a widely used alternative formulation of the boundary value problem (3) is the so-called regularized formulation of the Maxwell equations; see $[5,7-9]$. In fact, it is easily seen that the boundary value problem (3) is equivalent to the boundary value problem

$$
\begin{aligned}
\operatorname{curl} \operatorname{curl} \mathbf{E}-\operatorname{grad} \operatorname{div} \mathbf{E}-\alpha^{2} \mathbf{E}=\mathbf{f} & \text { in } \Omega, \\
\mathbf{E} \wedge \mathbf{n}=\mathbf{0} & \text { on } \Gamma, \\
\operatorname{div} \mathbf{E}=0 & \text { on } \Gamma,
\end{aligned}
$$

in the sense that the solution of (3) solves (8) and vice versa. We notice that the operator $\mathbf{E} \mapsto \operatorname{curl}$ curl $\mathbf{E}-\operatorname{grad} \operatorname{div} \mathbf{E}-\alpha^{2} \mathbf{E}$ is elliptic. The associated Hilbert space formulation for the boundary value problem (8) is as follows.

Find $\mathbf{u} \in \mathscr{H}_{0}$ (curl, div, $\Omega$ ) such that

$$
a(\mathbf{u}, \mathbf{v})=f(\mathbf{v}), \quad \forall \mathbf{v} \in \mathscr{H}_{0}(\operatorname{curl}, \operatorname{div}, \Omega),
$$

where

$$
\begin{aligned}
& a(\mathbf{u}, \mathbf{v}):=(\operatorname{curl} \mathbf{u}, \operatorname{curl} \mathbf{v})+(\operatorname{div} \mathbf{u}, \operatorname{div} \mathbf{v})-\alpha^{2}(\mathbf{u}, \mathbf{v}), \\
& f(\mathbf{v}):=(\mathbf{f}, \mathbf{v}), \\
& \mathscr{H}_{0}(\operatorname{curl}, \operatorname{div}, \Omega) \\
& \quad:=\left\{\mathbf{v} \in \mathscr{H}_{0}(\operatorname{curl}, \Omega): \operatorname{div} \mathbf{v} \in L_{2}(\Omega)\right\}, \\
& \|\mathbf{v}\|_{\mathscr{H}_{0}(\operatorname{curl}, \operatorname{div}, \Omega)} \\
& \quad:=\left(\|\mathbf{v}\|_{\left(L_{2}(\Omega)\right)^{3}}^{2}+\|\operatorname{curl} \mathbf{v}\|_{\left(L_{2}(\Omega)\right)^{3}}^{2}+\|\operatorname{div} \mathbf{v}\|_{L_{2}(\Omega)}^{2}\right)^{1 / 2} .
\end{aligned}
$$

The following theorem addresses the question of wellposedness of problem (9); see also [5, 9].

Theorem 2. Let $\Omega \subset \mathbb{R}^{3}$ be a bounded domain with at least Lipschitz continuous boundary $\Gamma$. Suppose $\alpha \neq 0$ and $\alpha^{2}$ is not an eigenvalue of the Dirichlet-Laplace operator on $\Omega$.
Then, for any $\mathbf{f} \in\left(L_{2}(\Omega)\right)^{3}$, there exists a unique solution $\mathbf{u} \in \mathscr{H}_{0}$ (curl, div, $\Omega$ ) of the variational problem (9). Moreover, the solution satisfies the estimate

$$
\begin{aligned}
& \|\mathbf{u}\|_{\left(L_{2}(\Omega)\right)^{3}}^{2}+\|\operatorname{curl} \mathbf{u}\|_{\left(L_{2}(\Omega)\right)^{3}}^{2}+\|\operatorname{div} \mathbf{u}\|_{L_{2}(\Omega)}^{2} \\
& \quad \leq C\|\mathbf{f}\|_{\left(L_{2}(\Omega)\right)^{3}}^{2} .
\end{aligned}
$$

The rest of this paper is dedicated to a rigorous regularity analysis of the solution of time-harmonic Maxwell equations in simply connected and bounded domains $\Omega \subset \mathbb{R}^{d}$, $d=2,3$, with Lipschitz boundary $\Gamma$ containing an isotropic and homogeneous medium subject to perfect conductor boundary conditions. We will systemically use the regularized formulation (8) and all derivatives should always be understood in the sense of distributions. First we state here one regularity result that is frequently quoted in the literature; see [21]. We will use the notation

$$
H_{N}(\Omega):=\left\{\mathbf{v} \in\left(H^{1}(\Omega)\right)^{d}: \mathbf{v} \wedge \mathbf{n}=0 \text { on } \Gamma\right\} .
$$

Theorem 3. Let $\Omega \subset \mathbb{R}^{d}$ be a bounded domain with boundary $\Gamma$. If $\Gamma$ is of class $\mathscr{C}^{1,1}$ or if $\Omega$ is a convex polygon in $\mathbb{R}^{2}$ or a convex polyhedron in $\mathbb{R}^{3}$, then the relation

$$
H_{N}(\Omega)=\mathscr{H}_{0}(\operatorname{curl}, \operatorname{div}, \Omega)
$$

holds and on these spaces the norms $\|\cdot\|_{\left(H^{1}(\Omega)\right)^{d}}$ and $\|\cdot\|_{\mathscr{H}(\mathrm{curl}, \mathrm{div}, \Omega)}$ are equivalent.

On the other hand, if $\Omega \subset \mathbb{R}^{d}$ is a nonconvex polygon or polyhedron, then the space $H_{N}(\Omega)$ is a proper closed subset of the space $\mathscr{H}_{0}$ (curl, div, $\Omega$ ) and the following result holds; see $[4,6]$.

Theorem 4. The space $\mathscr{H}_{0}(\mathrm{curl}, \mathrm{div}, \Omega)$ can be split as a direct sum of the form

$$
\mathscr{H}_{0}(\operatorname{curl}, \operatorname{div}, \Omega)=H_{N}(\Omega) \oplus \operatorname{grad} S,
$$

where $S$ denotes the space of functions spanned by the singular functions associated with the Dirichlet boundary value problem for the Laplace equation in $\Omega \subset \mathbb{R}^{d}$.

An immediate consequence of the regularity Theorems 3 and 4 is that, in nonconvex polygons or polyhedrons, the weak solution of the boundary value problem (8) does not belong to the space $H^{1}$ and can therefore not be approximated by means of the usual $H^{1}$-conforming nodal finite-element method.

\section{Corner and Edge Singularities for Maxwell's Equations}

In this section we formulate the main results of this paper. The proofs which are very lengthy in nature will be carried out in subsequent sections. 
3.1. Corner Singularities for Maxwell's Equations. Here we consider the electric field $\mathbf{u}$ of time-harmonic Maxwell's equations in a simply connected and bounded domain $\Omega \subset \mathbb{R}^{2}$ with Lipschitz boundary $\Gamma$, formally the variational solution of the boundary value problem

$$
\begin{aligned}
\operatorname{curl} \operatorname{curl} \mathbf{u}-\operatorname{grad} \operatorname{div} \mathbf{u}-\alpha^{2} \mathbf{u}=\mathbf{f} & \text { in } \Omega, \\
\mathbf{u} \wedge \mathbf{n}=0 & \text { on } \Gamma, \\
\operatorname{div} \mathbf{u}=0 & \text { on } \Gamma,
\end{aligned}
$$

where $\mathbf{f} \in\left(L_{2}(\Omega)\right)^{2}$ and the parameter $\alpha \neq 0$ are given.

Now, suppose that the boundary $\Gamma$ of $\Omega$ consists of finitely many disjoint analytic arcs $\Gamma_{j}, j=1, \ldots, J$, such that $\Gamma=\bigcup_{j=1}^{J} \bar{\Gamma}_{j}$, where the segments $\Gamma_{j}$ are numbered according to the positive orientation, that is, in anticlockwise direction. Let the endpoints of each $\Gamma_{j}$ be denoted by $A_{j}$ and let the solid angle at $A_{j}$ be denoted by $\omega_{j}$, where $0<\omega_{j}<$ $2 \pi$. We denote by $r_{j}$ and $\theta_{j}$ (resp., $x_{j}$ and $y_{j}$ ) local polar (resp., Cartesian) coordinates attached to the vertex $A_{j}$, such that

$$
\begin{aligned}
& x_{j}=r_{j} \cos \left(\theta_{j}\right), \\
& y_{j}=r_{j} \sin \left(\theta_{j}\right) ;
\end{aligned}
$$

that is, $\Gamma_{j}$ is supported by the line $\theta_{j}=\omega_{j}$ and $\Gamma_{j+1}$ is on the line $\theta_{j}=0$. Suppose that the domain $\Omega$ coincides near each singular point $A_{j}$ with a circular sector $\widetilde{\mathbb{K}}_{j}$ with radius $R_{j}$ and angle $\omega_{j}$; that is,

$$
\begin{aligned}
\widetilde{\mathbb{K}}_{j} & :=\left\{\left(x_{j}, y_{j}\right) \in \Omega: x_{j}=r_{j} \cos \left(\theta_{j}\right), y_{j}\right. \\
& \left.=r_{j} \sin \left(\theta_{j}\right), 0<r_{j}<R_{j}, 0<\theta_{j}<\omega_{j}\right\} .
\end{aligned}
$$

The boundary $\partial \widetilde{\mathbb{K}}_{j}$ will be represented subsequently as $\partial \widetilde{\mathbb{K}}_{j}=$ $\bar{\Gamma}_{j} \cup \bar{\Gamma}_{j+1} \cup \bar{\Gamma}_{j 0}$, where

$$
\begin{aligned}
\Gamma_{j} & :=\left\{\left(x_{j}, y_{j}\right): x_{j}=r_{j} \cos \left(\theta_{j}\right), y_{j}=r_{j} \sin \left(\theta_{j}\right), 0\right. \\
& \left.<r_{j}<R_{j}, \theta_{j}=\omega_{j}\right\}, \\
\Gamma_{j+1} & :=\left\{\left(x_{j}, y_{j}\right): x_{j}=r_{j} \cos \left(\theta_{j}\right), y_{j}\right. \\
& \left.=r_{j} \sin \left(\theta_{j}\right), 0<r_{j}<R_{j}, \theta_{j}=0\right\}, \\
\Gamma_{j 0} & :=\left\{\left(x_{j}, y_{j}\right): x_{j}=r_{j} \cos \left(\theta_{j}\right), y_{j}\right. \\
& \left.=r_{j} \sin \left(\theta_{j}\right), r_{j}=R_{j}, 0<\theta_{j}<\omega_{j}\right\} .
\end{aligned}
$$

We define with respect to the vertex $A_{j}$ a smooth truncation function $\eta_{j} \in \mathscr{D}(\bar{\Omega})$ which depends only on the distance $r_{j}$ from $A_{j}$ by

$$
\eta_{j}\left(x_{j}, y_{j}\right)=\eta_{j}\left(r_{j}\right):=\left\{\begin{aligned}
1, & \text { for } 0 \leq r_{j} \leq \frac{R_{j}}{3}, \\
0, & \text { for } r_{j} \geq \frac{2 R_{j}}{3}, \\
& 0 \leq \eta_{j}\left(r_{j}\right) \leq 1,
\end{aligned}\right.
$$

where $R_{j}$ is taken from (17); that is, $\operatorname{supp}\left(\eta_{j}\right) \subset \widetilde{\mathbb{K}}_{j}$. Furthermore, we define on each sector neighbourhood $\widetilde{\mathbb{K}}_{j}$ of the vertex $A_{j}$ the functions

$$
\begin{aligned}
f_{1 j}\left(x_{j}, y_{j}\right):= & \eta_{j}\left(f_{1}+\alpha^{2} u_{1}\right)-u_{1} \Delta_{x_{1} x_{2}} \eta_{j}-2 \nabla_{x_{1} x_{2}} \eta_{j} \\
& \cdot \nabla_{x_{1} x_{2}} u_{1} \\
f_{2 j}\left(x_{j}, y_{j}\right):= & \eta_{j}\left(f_{2}+\alpha^{2} u_{2}\right)-u_{2} \Delta_{x_{1} x_{2}} \eta_{j}-2 \nabla_{x_{1} x_{2}} \eta_{j} \\
& \cdot \nabla_{x_{1} x_{2}} u_{2},
\end{aligned}
$$

where $\mathbf{f}=\left(f_{1}, f_{2}\right), \mathbf{u}=\left(u_{1}, u_{2}\right)$ and the parameter $\alpha$ are taken from (15), $\eta_{j}$ is as defined in (19), and

$$
\begin{aligned}
\Delta_{x_{1} x_{2}} & :=\left(\frac{\partial^{2}}{\partial x_{1}^{2}}+\frac{\partial^{2}}{\partial x_{2}^{2}}\right) ; \\
\nabla_{x_{1} x_{2}} & :=\left(\frac{\partial}{\partial x_{1}}, \frac{\partial}{\partial x_{2}}\right) .
\end{aligned}
$$

Our main result on corner singularity is the following.

Theorem 5. For each $\mathbf{f}=\left(f_{1}, f_{2}\right) \in\left(L_{2}(\Omega)\right)^{2}$ and $\alpha \neq 0$, let $\mathbf{u} \in \mathscr{H}_{0}$ (curl, div, $\Omega$ ) be the variational solution of the boundary value problem (15). Let $\lambda_{j k}:=k \pi / \omega_{j}, k \in \mathbb{N}, \omega_{j} \neq \pi$, $j=1, \ldots$, J. If $\lambda_{j k} \neq 2, k \in \mathbb{N}, j=1, \ldots, J$, then there exist coefficients $\gamma_{j k}$ such that the solution $\mathbf{u}$ can be split as a sum in the form

$$
\begin{aligned}
\mathbf{u}=\mathbf{w}+\sum_{\substack { j=1 \\
\begin{subarray}{c}{0<\lambda_{j k}<2 \\
\lambda_{j k} \neq 1{ j = 1 \\
\begin{subarray} { c } { 0 < \lambda _ { j k } < 2 \\
\lambda _ { j k } \neq 1 } }\end{subarray}} \gamma_{j k} r_{j}^{\lambda_{j k}-1}\left(\sin \left(\left(\lambda_{j k}-1\right) \theta_{j}\right),\right. \\
\left.\quad \cos \left(\left(\lambda_{j k}-1\right) \theta_{j}\right)\right)
\end{aligned}
$$

with $\mathbf{w}=\left(w_{1}, w_{2}\right) \in\left(H^{2}(\Omega)\right)^{2}$. The coefficients $\gamma_{j k}$ of the asymptotic expansion (22) are given explicitly by the formula

$$
\begin{aligned}
& \gamma_{j k}:=-\frac{R_{j}^{2\left(1-\lambda_{j k}\right)}}{2 \omega_{j}\left(\lambda_{j k}-1\right)} \\
& \cdot \int_{\widetilde{\mathbb{K}}_{j}}\left\{f_{1 j}\left(x_{j}, y_{j}\right) \sin \left(\left(\lambda_{j k}-1\right) \theta_{j}\right)\right. \\
&\left.+f_{2 j}\left(x_{j}, y_{j}\right) \cos \left(\left(\lambda_{j k}-1\right) \theta_{j}\right)\right\} r_{j}^{\lambda_{j k}-1} d x_{1} d x_{2},
\end{aligned}
$$


where the function $\mathbf{f}_{j}=\left(f_{1 j}, f_{2 j}\right)$ is defined in (20). The constants $R_{j}$ and $\omega_{j}$ and the local Cartesian and polar coordinates $x_{j}, y_{j}$ and $r_{j}, \theta_{j}$ are as specified in (17) and (16). Moreover, there exists a constant $C>0$ independent of $\mathbf{f}$ and $\mathbf{u}$ such that

$$
\left|\gamma_{j k}\right|+\|\mathbf{w}\|_{\left(H^{2}(\Omega)\right)^{2}} \leq C\|\mathbf{f}\|_{\left(L_{2}(\Omega)\right)^{2}} .
$$

The proof of Theorem 5 will be carried out in Section 4; see Theorem 9.

3.2. Edge Singularities for Maxwell's Equations. Let $Q \subset \mathbb{R}^{3}$ be a simply connected and bounded domain with Lipschitz boundary $\partial Q$. For $\mathbf{f} \in\left(L_{2}(Q)\right)^{3}$ and $\alpha \neq 0$, we consider the variational solution $\mathbf{u}=\left(u_{1}, u_{2}, u_{3}\right) \in \mathscr{H}_{0}(\operatorname{curl}, \operatorname{div}, Q)$ of the boundary value problem

$$
\begin{aligned}
\operatorname{curl} \operatorname{curl} \mathbf{u}-\operatorname{grad} \operatorname{div} \mathbf{u}-\alpha^{2} \mathbf{u}=\mathbf{f} & \text { in } Q, \\
\mathbf{u} \wedge \mathbf{n}=\mathbf{0} & \text { on } \partial Q, \\
\operatorname{div} \mathbf{u}=0 & \text { on } \partial Q .
\end{aligned}
$$

Since we are interested only in the asymptotic behaviour of the solution $\mathbf{u}$ near straight edges of the domain $Q$, we can assume, without loss of generality, that the domain $Q$ is a prismatic cylinder; that is, it has the form $Q=\Omega \times(0, l)$ with a real constant $l>0$ and a bounded domain $\Omega \subset \mathbb{R}^{2}$ with piecewise smooth boundary $\Gamma$ and such that for each $\left(x_{1}, x_{2}, x_{3}\right) \in Q,\left(x_{1}, x_{2}\right) \in \Omega$, and $x_{3} \in(0, l)$. In this way we can use the same notations as in Section 3.1 for $\Omega$. In particular, the edges of the domain $Q$ are $E_{j}=A_{j} \times(0, l)$ and the measure of the interior angle along the edge $E_{j}$ is $\omega_{j}, j=1, \ldots, J$. We associate with each edge $E_{j}$ a wedge $G_{j}=\widetilde{\mathbb{K}}_{j} \times(0, l)$, where $\widetilde{\mathbb{K}}_{j} \subset \Omega$ is as defined in (17). Further we introduce on $G_{j}$ the functions

$$
\begin{aligned}
f_{1 j}^{*}\left(x_{j}, y_{j}, x_{3}\right) & \\
:= & \eta_{j}\left(f_{1}+\alpha^{2} u_{1}\right)-u_{1} \Delta_{x_{1} x_{2}} \eta_{j}-2 \nabla_{x_{1} x_{2}} \eta_{j} \cdot \nabla_{x_{1} x_{2}} u_{1} \\
& -\frac{n \pi}{l} \frac{\left(\eta_{j} u_{1}\right)}{r_{j}} \\
& -\frac{2 n \pi}{l}\left(\cos \theta_{j} \frac{\partial\left(\eta_{j} u_{1}\right)}{\partial x_{1}}+\sin \theta_{j} \frac{\partial\left(\eta_{j} u_{1}\right)}{\partial x_{2}}\right) \\
& -\left(\frac{n \pi}{l}\right)^{2}\left(\eta_{j} u_{1}\right), \\
f_{2 j}^{*}( & \left.x_{j}, y_{j}, x_{3}\right) \\
:= & \eta_{j}\left(f_{2}+\alpha^{2} u_{2}\right)-u_{2} \Delta_{x_{1} x_{2}} \eta_{j}-2 \nabla_{x_{1} x_{2}} \eta_{j} \cdot \nabla_{x_{1} x_{2}} u_{2} \\
& -\frac{n \pi}{l} \frac{\left(\eta_{j} u_{2}\right)}{r_{j}}
\end{aligned}
$$

$$
\begin{aligned}
& -\frac{2 n \pi}{l}\left(\cos \theta_{j} \frac{\partial\left(\eta_{j} u_{2}\right)}{\partial x_{1}}+\sin \theta_{j} \frac{\partial\left(\eta_{j} u_{2}\right)}{\partial x_{2}}\right) \\
& -\left(\frac{n \pi}{l}\right)^{2}\left(\eta_{j} u_{1}\right), \\
f_{3 j}^{*}\left(x_{j}, y_{j}, x_{3}\right) & \\
:= & \eta_{j}\left(f_{3}+\alpha^{2} u_{3}\right)-u_{3} \Delta_{x_{1} x_{2}} \eta_{j}-2 \nabla_{x_{1} x_{2}} \eta_{j} \cdot \nabla_{x_{1} x_{2}} u_{3} \\
& -\frac{n \pi}{l} \frac{\left(\eta_{j} u_{3}\right)}{r_{j}} \\
& -\frac{2 n \pi}{l}\left(\cos \left(\theta_{j}\right) \frac{\partial\left(\eta_{j} u_{3}\right)}{\partial x_{1}}+\sin \left(\theta_{j}\right) \frac{\partial\left(\eta_{j} u_{3}\right)}{\partial x_{2}}\right) \\
& -\left(\frac{2 n \pi}{l}\right)^{2}\left(\eta_{j} u_{3}\right),
\end{aligned}
$$

where the functions $\mathbf{f}=\left(f_{1}, f_{2}, f_{3}\right), \mathbf{u}=\left(u_{1}, u_{2}, u_{3}\right)$, and the parameter $\alpha$ are taken from (25), $\eta_{j}$ is from (19), and $\Delta_{x_{1} x_{2}}$, $\nabla_{x_{1} x_{2}}$ are as defined in (21). Obviously $\mathbf{f}_{j}^{*}=\left(f_{1 j}^{*}, f_{2 j}^{*}, f_{3 j}^{*}\right) \epsilon$ $\left(L_{2}\left(G_{j}\right)\right)^{3}$.

Our main result on edge singularity is the following.

Theorem 6. For $\mathbf{f} \in\left(L_{2}(Q)\right)^{3}$ and $\alpha \neq 0$, let $\mathbf{u} \in \mathscr{H}_{0}$ (curl, $\operatorname{div}, Q)$ be the variational solution of the boundary value problem (25). Let $\lambda_{j k}:=k \pi / \omega_{j}, k \in \mathbb{N}, \omega_{j} \neq \pi, j=1, \ldots$, J. If $\lambda_{j k} \neq 2, k \in \mathbb{N}, j=1, \ldots, J$, then there exist unique functions $\Psi_{j k} \in H^{\lambda_{j k}}(0, l)$ and $\Psi_{j} \in H^{1-\lambda_{j 1}}(0, l)$ such that the solution $\mathbf{u} \in \mathscr{H}_{0}$ (curl, div, Q) can be split into a regular and a singular part in the form

$$
\begin{aligned}
\mathbf{u}= & \left(w_{1}, w_{2}, w_{3}\right)+\left(s_{1}, s_{2}, s_{3}\right), \\
\mathbf{w} \in & \left(H^{2}(Q)\right)^{3}, \\
s_{1}\left(x_{1}, x_{2}, x_{3}\right) & \\
:= & \sum_{j=1}^{J} \sum_{0<\lambda_{j k}<2} \Psi_{j k}\left(x_{3}\right) \\
& * T_{j}\left(r_{j}, x_{3}\right) r_{j}^{\lambda_{j k}-1} \sin \left(\left(\lambda_{j k}-1\right) \theta_{j}\right), \\
s_{2}\left(x_{1}, x_{2}, x_{3}\right) & :=\sum_{j=1}^{J} \sum_{0<\lambda_{k}<2} \Psi_{j k}\left(x_{3}\right) \\
& * T_{j}\left(r_{j}, x_{3}\right) r_{j}^{\lambda_{j k}-1} \cos \left(\left(\lambda_{j k}-1\right) \theta_{j}\right),
\end{aligned}
$$




$$
\begin{aligned}
& s_{3}\left(x_{1}, x_{2}, x_{3}\right) \\
& :=\sum_{j=1}^{J} \Psi_{j}\left(x_{3}\right) * T_{3 j}\left(r_{j}, x_{3}\right) r_{j}^{\lambda_{j 1}} \sin \left(\lambda_{j 1} \theta_{j}\right) \\
& \text { if } 0<\lambda_{j 1}<1 .
\end{aligned}
$$

The functions $T_{j}$ and $T_{3 j}$ are fixed kernels defined by

$$
\begin{aligned}
T_{j}\left(r_{j}, x_{3}\right) & :=\sum_{n=1}^{\infty} \mathrm{e}^{-(n \pi / l) r_{j}} \sin \frac{n \pi x_{3}}{l}, \\
T_{3 j}\left(r_{j}, x_{3}\right) & :=\sum_{n=1}^{\infty} \mathrm{e}^{-(n \pi / l) r_{j}} \cos \frac{n \pi x_{3}}{l} .
\end{aligned}
$$

The coefficients $\Psi_{j k}$ and $\Psi_{j}$ of the asymptotic expansion (27) can be expressed in Fourier series in the form

$$
\begin{aligned}
\Psi_{j k}\left(x_{3}\right) & :=\sum_{n=1}^{\infty} \gamma_{j k n} \sin \frac{n \pi x_{3}}{l}, \\
\Psi_{j}\left(x_{3}\right) & :=\frac{1}{2} \gamma_{j 0}+\sum_{n=1}^{\infty} \gamma_{j n} \cos \frac{n \pi x_{3}}{l},
\end{aligned}
$$

where the Fourier coefficients $\gamma_{j k n}$ and $\gamma_{j n}$ are given explicitly by the formulas

$$
\begin{aligned}
& \gamma_{j k n}:=-\frac{R_{j}^{2-2 \lambda_{j k}}}{l \omega_{j}\left(\lambda_{j k}-1\right)} \int_{G_{j}} \sin \left(\frac{n \pi x_{3}}{l}\right) \\
& \cdot \mathrm{e}^{(n \pi / l) r_{j}}\left\{f_{1 j}^{*}\left(x_{j}, y_{j}, x_{3}\right) \sin \left(\left(\lambda_{j k}-1\right) \theta_{j}\right)\right. \\
&\left.+f_{2 j}^{*}\left(x_{j}, y_{j}, x_{3}\right) \cos \left(\left(\lambda_{j k}-1\right) \theta_{j}\right)\right\} \\
& \cdot r_{j}^{\lambda_{j k}-1} d x_{1} d x_{2} d x_{3}, \\
& \gamma_{j n}:=\frac{2}{l \omega_{j} \lambda_{j 1}} \int_{G_{j}} \cos \left(\frac{n \pi x_{3}}{l}\right) \mathrm{e}^{(n \pi / l) r_{j}} f_{3 j}^{*}\left(x_{j}, y_{j}, x_{3}\right) \\
& \cdot \sin \left(\lambda_{j 1} \theta_{j}\right) r_{j}^{-\lambda_{j 1}} d x_{1} d x_{2} d x_{3} .
\end{aligned}
$$

Here, the function $\mathbf{f}_{j}^{*}=\left(f_{1 j}^{*}, f_{2 j}^{*}, f_{3 j}^{*}\right)$ is as defined in (26). The constants $R_{j}$ and $\omega_{j}$ and the local Cartesian and polar coordinates $x_{j}, y_{j}, r_{j}$, and $\theta_{j}$ are as defined in (17) and (16). In (27) the symbol " $*$ " denotes convolution product in the variable $x_{3}$; that is,

$$
\begin{aligned}
\Psi_{j k}\left(x_{3}\right) * T_{j}\left(r_{j}, x_{3}\right):= & \sum_{n=1}^{\infty} \gamma_{n j k} \mathrm{e}^{-(n \pi / l) r_{j}} \sin \frac{n \pi x_{3}}{l}, \\
\Psi_{j}\left(x_{3}\right) * T_{3 j}\left(r_{j}, x_{3}\right):= & \frac{1}{2} \gamma_{j 0} \\
& +\sum_{n=1}^{\infty} \gamma_{j n} \mathrm{e}^{-(n \pi / l) r_{j}} \cos \frac{n \pi x_{3}}{l} .
\end{aligned}
$$

The proof of Theorem 6 will be carried out in Section 5; see Theorem 24.

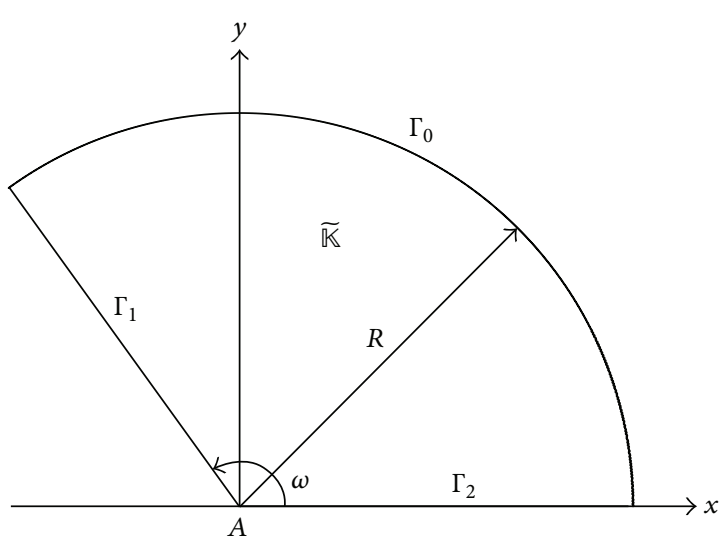

Figure 1: A circular sector.

\section{Maxwell's Equations in Two-Dimensional Domains with Corners}

In this section, we consider in greater detail the structure of the solution of the Maxwell equations (15) in twodimensional domains with corners and show how the results of Theorem 5 are derived.

4.1. Maxwell's Equations in a Bounded Sector. For purely mathematical reasons we consider first a slightly modified boundary value problem for the Maxwell equations in a circular sector; see Figure 1. The results of this subsection are largely found in Nkemzi [13] and will be kept very brief.

Let $\widetilde{\mathbb{K}}$ denote a circular sector in $\mathbb{R}^{2}$ with radius $R$, interior angle $\omega \neq \pi$, and boundary $\partial \widetilde{\mathbb{K}}=\bar{\Gamma}_{0} \cup \bar{\Gamma}_{1} \cup \bar{\Gamma}_{2}$; see Figure 1 . We assume that the Cartesian coordinate system of $x_{1}$ and $x_{2}$ is positioned such that the vertex $A$ of $\widetilde{\mathbb{K}}$ coincides with the origin and the side $\Gamma_{2}$ is supported by the $x_{1}$-axis.

For $\widetilde{\mathbf{f}}=\left(f_{1}, f_{2}\right) \in L_{2}(\widetilde{\mathbb{K}})^{2}$, we consider the unique weak solution $\widetilde{\mathbf{u}}=\left(u_{1}, u_{2}\right) \in \mathscr{H}_{0}(\operatorname{curl}, \operatorname{div}, \widetilde{\mathbb{K}})$ of the boundary value problem

$$
\begin{aligned}
\operatorname{curlcurl} \widetilde{\mathbf{u}}-\operatorname{grad} \operatorname{div} \widetilde{\mathbf{u}} & =\widetilde{\mathbf{f}} & & \text { in } \widetilde{\mathbb{K}}, \\
\widetilde{\mathbf{u}} \wedge \mathbf{n} & =0 & & \text { on } \Gamma_{1} \cup \Gamma_{2}, \\
\operatorname{div} \widetilde{\mathbf{u}} & =0 & & \text { on } \Gamma_{1} \cup \Gamma_{2}, \\
\widetilde{\mathbf{u}} & =0 & & \text { on } \Gamma_{0} .
\end{aligned}
$$

Local polar coordinates $r$ and $\theta$ in $\widetilde{\mathbb{K}}$ with respect to the vertex $A$ are related to the Cartesian coordinates $x_{1}$ and $x_{2}$; namely,

$$
\begin{aligned}
& x_{1}=r \cos \theta, \\
& x_{2}=r \sin \theta,
\end{aligned}
$$

$$
0<r<R, 0<\theta<\omega .
$$


Accordingly, the sector domain $\widetilde{K}$ is transformed by the oneto-one mapping into the rectangle

$$
\mathbb{K}=\left\{(r, \theta): 0<r<R_{0}, 0<\theta<\omega\right\}
$$

in the polar coordinate system. By the transformation (33), each function $\widetilde{u}=\widetilde{u}\left(x_{1}, x_{2}\right)$ defined on $\widetilde{\mathbb{K}}$ is mapped uniquely to some function $u=u(r, \theta)$ defined on $\mathbb{K}$ by

$$
u(r, \theta)=\widetilde{u}(r \cos \theta, r \sin \theta) .
$$

Similarly, each vector field $\widetilde{\mathbf{u}}=\left(u_{1}\left(x_{1}, x_{2}\right), u_{2}\left(x_{1}, x_{2}\right)\right)$ defined on $\widetilde{\mathbb{K}}$ is mapped uniquely to some vector field $\mathbf{u}=$ $\left(u_{r}(r, \theta), u_{\theta}(r, \theta)\right)$ defined on $\mathbb{K}$ by

$$
\begin{aligned}
& u_{r}(r, \theta)=u_{1}\left(x_{1}, x_{2}\right) \cos \theta+u_{2}\left(x_{1}, x_{2}\right) \sin \theta, \\
& u_{\theta}(r, \theta)=-u_{1}\left(x_{1}, x_{2}\right) \sin \theta+u_{2}\left(x_{1}, x_{2}\right) \cos \theta .
\end{aligned}
$$

The boundary value problem (32) can be solved explicitly. In fact, we have the following result which can be verified by direct substitution.

Lemma 7. The weak solution $\widetilde{\mathbf{u}}=\mathbf{u}=\left(u_{r}(r, \theta), u_{\theta}(r, \theta)\right)$ of the boundary value problem (32) can be represented in Fourier series in the form

$$
\begin{aligned}
& u_{r}(r, \theta)=\sum_{k=1}^{\infty} u_{r k}(r) \sin \lambda_{k} \theta, \\
& u_{\theta}(r, \theta)=\sum_{k=1}^{\infty} u_{r k}(r) \cos \lambda_{k} \theta,
\end{aligned}
$$

where the Fourier coefficients $\left\{\mathbf{u}_{k}=\left(u_{r k}, u_{\theta k}\right): k \in \mathbb{N}\right\}$ satisfy the relations

$$
\begin{aligned}
& u_{r k}(r)=-\frac{r^{\lambda_{k}-1}}{4\left(\lambda_{k}-1\right) R^{2 \lambda_{k}-2}} \\
& \cdot \int_{0}^{R}\left(f_{r k}(\tau)+f_{\theta k}(\tau)\right) \tau^{\lambda_{k}} d \tau+\frac{r^{\lambda_{k}+1}}{4\left(\lambda_{k}+1\right) R^{2 \lambda_{k}+2}} \\
& \cdot \int_{0}^{R}\left(-f_{r k}(\tau)+f_{\theta k}(\tau)\right) \tau^{\lambda_{k}+2} d \tau+\frac{r^{-\left(\lambda_{k}+1\right)}}{4\left(\lambda_{k}+1\right)} \\
& \cdot \int_{0}^{r}\left(f_{r k}(\tau)-f_{\theta k}(\tau)\right) \tau^{\lambda_{k}+2} d \tau+\frac{r^{1-\lambda_{k}}}{4\left(\lambda_{k}-1\right)} \\
& \cdot \int_{0}^{r}\left(f_{r k}(\tau)+f_{\theta k}(\tau)\right) \tau^{\lambda_{k}} d \tau+\frac{r^{\lambda_{k}-1}}{4\left(\lambda_{k}-1\right)} \\
& \cdot \int_{r}^{R}\left(f_{r k}(\tau)+f_{\theta k}(\tau)\right) \tau^{2-\lambda_{k}} d \tau-\frac{r^{\lambda_{k}+1}}{4\left(\lambda_{k}+1\right)} \\
& \cdot \int_{r}^{R}\left(-f_{r k}(\tau)+f_{\theta k}(\tau)\right) \tau^{-\lambda_{k}} d \tau, \quad \lambda_{k} \neq 1,
\end{aligned}
$$

$$
\begin{aligned}
& u_{\theta k}(r)=-\frac{r^{\lambda_{k}-1}}{4\left(\lambda_{k}-1\right) R^{2 \lambda_{k}-2}} \\
& \cdot \int_{0}^{R}\left(f_{r k}(\tau)+f_{\theta k}(\tau)\right) \tau^{\lambda_{k}} d \tau-\frac{r^{\lambda_{k}+1}}{4\left(\lambda_{k}+1\right) R^{2 \lambda_{k}+2}} \\
& \cdot \int_{0}^{R}\left(-f_{r k}(\tau)+f_{\theta k}(\tau)\right) \tau^{\lambda_{k}+2} d \tau-\frac{r^{-\left(\lambda_{k}+1\right)}}{4\left(\lambda_{k}+1\right)} \\
& \cdot \int_{0}^{r}\left(f_{r k}(\tau)-f_{\theta k}(\tau)\right) \tau^{\lambda_{k}+2} d \tau+\frac{r^{1-\lambda_{k}}}{4\left(\lambda_{k}-1\right)} \\
& \cdot \int_{0}^{r}\left(f_{r k}(\tau)+f_{\theta k}(\tau)\right) \tau^{\lambda_{k}} d \tau+\frac{r^{\lambda_{k}-1}}{4\left(\lambda_{k}-1\right)} \\
& \cdot \int_{r}^{R}\left(f_{r k}(\tau)+f_{\theta k}(\tau)\right) \tau^{2-\lambda_{k}} d \tau+\frac{r^{\lambda_{k}+1}}{4\left(\lambda_{k}+1\right)} \\
& \cdot \int_{r}^{R}\left(-f_{r k}(\tau)+f_{\theta k}(\tau)\right) \tau^{-\lambda_{k}} d \tau, \quad \lambda_{k} \neq 1 .
\end{aligned}
$$

Using the explicit representation formulas (37)-(38) for the solution of the boundary value problem (32) and taking into account relation (36) one can derive various regularity properties for the solution.

The main result of this subsection is the following; see, for example, $[13,15,16]$, for the proof.

Theorem 8. Let $\widetilde{\mathbb{K}}$ be a circular sector with angle $\omega \in(0,2 \pi)$, $\omega \neq \pi$. Let $\lambda_{k}=k \pi / \omega, k \in \mathbb{N}$. Then for each $\widetilde{\mathbf{f}} \in\left(L_{2}(\widetilde{\mathbb{K}})\right)^{2}$ the solution $\widetilde{\mathbf{u}} \in \mathscr{H}_{0}(\mathrm{curl}$, div, $\widetilde{K})$ of the boundary value problem (32) has the following additional regularity properties:

(a) There exists a constant $C>0$ independent of $\widetilde{\mathbf{f}}$ and $\widetilde{\mathbf{u}}$ such that the

$$
\|\operatorname{div} \widetilde{\mathbf{u}}\|_{L_{2}(\widetilde{\mathbb{K}})} \leq C\|\widetilde{\mathbf{f}}\|_{\left(L_{2}(\mathbb{K})\right)^{2}} .
$$

That is, the condition $\operatorname{div} \widetilde{\mathbf{u}} \in L_{2}(\widetilde{\mathbb{K}})$ is always satisfied.

(b) If $\lambda_{k}>1, k \in \mathbb{N}$ (i.e., $\widetilde{\mathbb{K}}$ is convex), then $\widetilde{\mathbf{u}} \in\left(H^{1}(\widetilde{\mathbb{K}})\right)^{2}$ and there exists a constant $C>0$ independent of $\widetilde{\mathbf{f}}$ and $\widetilde{\mathbf{u}}$ such that

$$
\|\widetilde{\mathbf{u}}\|_{H^{1}(\widetilde{\mathbb{K}})^{2}} \leq C\|\widetilde{\mathbf{f}}\|_{\left(L_{2}(\widetilde{\mathbb{K}})\right)^{2}} .
$$

(c) If $\lambda_{k}>2, k \in \mathbb{N}$ (i.e., $\left.0<\omega<\pi / 2\right)$, then $\widetilde{\mathbf{u}} \in\left(H^{2}(\widetilde{\mathbb{K}})\right)^{2}$ and there exists a constant $C>0$ independent of $\widetilde{\mathbf{f}}$ and $\widetilde{\mathbf{u}}$ such that

$$
\|\widetilde{\mathbf{u}}\|_{H^{2}(\widetilde{\mathbb{K}})^{2}} \leq C\|\widetilde{\mathbf{f}}\|_{\left(L_{2}(\widetilde{\mathbb{K}})\right)^{2}} .
$$


(d) If $\lambda_{k} \neq 2, k \in \mathbb{N}$, then $\tilde{\mathbf{u}}$ can be split into a regular and a singular part in the form

$\widetilde{\mathbf{u}}$

$$
\begin{aligned}
=\widetilde{\mathbf{w}} & \\
& +\sum_{\substack{0<\lambda_{k}<2 \\
\lambda_{k} \neq 1}} \gamma_{k} r^{\lambda_{k}-1}\left(\sin \left(\left(\lambda_{k}-1\right) \theta\right), \cos \left(\left(\lambda_{k}-1\right) \theta\right)\right)
\end{aligned}
$$

$$
\text { with } \widetilde{\mathbf{w}} \in\left(H^{2}(\widetilde{\mathbb{K}})\right)^{2} \text {. }
$$

The coefficients $\gamma_{k}$ are given explicitly by the formula

$$
\begin{aligned}
\gamma_{k} & :=-\frac{R_{0}^{2\left(1-\lambda_{k}\right)}}{2 \omega\left(\lambda_{k}-1\right)} \int_{\widetilde{\mathbb{K}}}\left(f_{1}\left(x_{1}, x_{2}\right) \sin \left(\left(\lambda_{k}-1\right) \theta\right)\right. \\
& \left.+f_{2}\left(x_{1}, x_{2}\right) \cos \left(\left(\lambda_{k}-1\right) \theta\right)\right) r^{\lambda_{k}-1} d x_{1} d x_{2} .
\end{aligned}
$$

Moreover, there exists a constant $C>0$ independent of $\widetilde{\mathbf{f}}$ such that

$$
\left|\gamma_{k}\right|+\|\widetilde{\mathbf{w}}\|_{\left(H^{2}(\widetilde{\mathbb{K}})\right)^{2}} \leq C\|\widetilde{\mathbf{f}}\|_{\left(L_{2}(\widetilde{\mathbb{K}})\right)^{2}} .
$$

4.2. Maxwell's Equations in Plane Domains with Corners. We can now make definite statements on the regularity properties of the solution $\mathbf{u} \in \mathscr{H}_{0}$ (curl, div, $\Omega$ ) of the Maxwell boundary value problem (15) in bounded domains $\Omega \subset \mathbb{R}^{2}$ with piecewise smooth boundary $\Gamma$. We will use the same notations as in Section 3.1.

Let $\mathbf{u} \in \mathscr{H}_{0}$ (curl, div, $\Omega$ ) be the solution of (15). Then the function $\mathbf{u}_{j}:=\eta_{j} \mathbf{u}$, where $\eta_{j}$ is the smooth truncation function from (19), belongs to the space $\mathscr{H}_{0}$ (curl, div, $\widetilde{\mathbb{K}}_{j}$ ) and is the unique weak solution of the boundary value problem

$$
\begin{aligned}
& \operatorname{curl} \operatorname{curl} \mathbf{u}_{j}-\operatorname{grad} \operatorname{div} \mathbf{u}_{j}=\mathbf{f}_{j} \quad \text { in } \widetilde{\mathbb{K}}_{j}, \\
& \mathbf{u}_{j} \wedge \mathbf{n}=0 \quad \text { on } \Gamma_{j} \cup \Gamma_{j+1} \text {, } \\
& \operatorname{div} \mathbf{u}_{j}=0 \quad \text { on } \Gamma_{j} \cup \Gamma_{j+1} \text {, } \\
& \mathbf{u}_{j}=\mathbf{0} \text { on } \Gamma_{j 0},
\end{aligned}
$$

where the function $\mathbf{f}_{j}=\left(f_{1 j}\left(x_{j}, y_{j}\right), f_{2 j}\left(x_{j}, y_{j}\right)\right) \in\left(L_{2}\left(\widetilde{\mathbb{K}}_{j}\right)\right)^{2}$ is as defined in (20).

We observe that problems (45) and (32) are similar and therefore their solutions have the same regularity properties as described in Theorem 8 . On the other hand, the solution $\mathbf{u}_{j} \in \mathscr{H}_{0}\left(\right.$ curl, div, $\widetilde{\mathbb{K}}_{j}$ ) of problem (45) coincides near the vertex $A_{j}$ of $\Omega$ to the solution $\mathbf{u} \in \mathscr{H}_{0}(\operatorname{curl}, \operatorname{div}, \Omega$ ) of problem (15). Thus the two solutions have the same asymptotic behaviour near the vertex $A_{j}$. Taking into consideration the fact that singularity is a local property and the technique for coupling local and global regularity properties (see $[2,18]$ ), we obtain directly from Theorem 8 the following properties for the solution $\mathbf{u} \in \mathscr{H}_{0}(\operatorname{curl}, \operatorname{div}, \Omega)$ of problem (15).
Theorem 9. For each $\mathbf{f} \in\left(L_{2}(\Omega)\right)^{2}$, let $\mathbf{u} \in \mathscr{H}_{0}$ (curl, div, $\Omega$ ) be the solution of the boundary value problem (15). Let $\lambda_{j k}=$ $k \pi / \omega_{j}, k \in \mathbb{N}, j=1, \ldots, J$, and $\omega_{j} \neq \pi$. Then the solution $\mathbf{u}$ has the following additional regularity properties:

(a) $\operatorname{div} \mathbf{u} \in L_{2}(\Omega)$ and there exists a constant $C>0$ independent of $\mathbf{f}$ and $\mathbf{u}$ such that

$$
\|\operatorname{div} \mathbf{u}\|_{L_{2}(\Omega)} \leq C\|\mathbf{f}\|_{\left(L_{2}(\Omega)\right)^{2}} .
$$

(b) If $\lambda_{j k}>1, k \in \mathbb{N}, j=1, \ldots, J$ (i.e., $\Omega$ is convex), then $\mathbf{u} \in H_{N}^{1}(\Omega)$ and there exists a constant $C>0$ independent of $\mathbf{f}$ and $\mathbf{u}$ such that

$$
\|\mathbf{u}\|_{H^{1}(\Omega)^{2}} \leq C\|\mathbf{f}\|_{\left(L_{2}(\Omega)\right)^{2}} .
$$

(c) If $\lambda_{j k}>2, k \in \mathbb{N}, j=1, \ldots, J$ (i.e., $0<\omega_{j}<\pi / 2$ for all $j)$, then $\mathbf{u} \in\left(H^{2}(\Omega)\right)^{2}$ and there exists a constant $C>0$ independent of $\mathbf{f}$ and $\mathbf{u}$ such that

$$
\|\mathbf{u}\|_{H^{2}(\Omega)^{2}} \leq C\|\mathbf{f}\|_{\left(L_{2}(\Omega)\right)^{2}} .
$$

(d) If $\lambda_{j k} \neq 2, k \in \mathbb{N}, j=1, \ldots, J$, then the solution $\mathbf{u}$ can be split into a regular and a singular part in the form

$$
\begin{aligned}
& \mathbf{u}=\mathbf{w}+\sum_{\substack { j=1 \\
\begin{subarray}{c}{0<\lambda_{j k}<2 \\
\lambda_{j k} \neq 1{ j = 1 \\
\begin{subarray} { c } { 0 < \lambda _ { j k } < 2 \\
\lambda _ { j k } \neq 1 } }\end{subarray}} \gamma_{j k} r_{j}^{\lambda_{j k}-1} \\
& \cdot\left(\sin \left(\left(\lambda_{j k}-1\right) \theta_{j}\right), \cos \left(\left(\lambda_{j k}-1\right) \theta_{j}\right)\right), \\
& \mathbf{w} \in\left(H^{2}(\Omega)\right)^{2} .
\end{aligned}
$$

The coefficients $\gamma_{j k}$ are given explicitly by the formula

$$
\begin{aligned}
& \gamma_{j k}:=-\frac{R_{j}^{2\left(1-\lambda_{j k}\right)}}{2 \omega_{j}\left(\lambda_{j k}-1\right)} \\
& \cdot \int_{\widetilde{\mathbb{K}}_{j}}\left(f_{1 j}\left(x_{j}, y_{j}\right) \sin \left(\left(\lambda_{j k}-1\right) \theta_{j}\right)\right. \\
&\left.+f_{2 j}\left(x_{j}, y_{j}\right) \cos \left(\left(\lambda_{j k}-1\right) \theta_{j}\right)\right) r_{j}^{\lambda_{j k}-1} d x_{1} d x_{2},
\end{aligned}
$$

where $\mathbf{f}_{j}=\left(f_{1 j}\left(x_{j}, y_{j}\right), f_{2 j}\left(x_{j}, y_{j}\right)\right)$ is defined in (20) and the local Cartesian coordinates $x_{j}$ and $y_{j}$ are defined in (16). Moreover, there exists a constant $C>0$ independent of $\mathbf{f}$ such that

$$
\left|\gamma_{j k}\right|+\|\mathbf{w}\|_{\left(H^{2}(\Omega)\right)^{2}} \leq C\|\mathbf{f}\|_{\left(L_{2}(\Omega)\right)^{2}} .
$$

4.3. Some Auxiliary Boundary Value Problems with a Parameter. Let $\tilde{\mathbf{f}}=\left(f_{1}\left(x_{1}, x_{2}\right), f_{2}\left(x_{1}, x_{2}\right)\right) \in\left(L_{2}(\widetilde{\mathbb{K}})\right)^{2}$ and $\tilde{f}=$ $\tilde{f}\left(x_{1}, x_{2}\right) \in L_{2}(\widetilde{\mathbb{K}})$ be given functions. We consider in this subsection the following boundary value problems with 
parameter on the sector domain $\widetilde{\mathbb{K}} \subset \mathbb{R}^{2}$ (see Figure 1), formulated in polar coordinates:

$$
\begin{aligned}
& -\frac{\partial^{2} u_{r}}{\partial r^{2}}-\frac{1}{r} \frac{\partial u_{r}}{\partial r}-\frac{1}{r^{2}} \frac{\partial^{2} u_{r}}{\partial \theta^{2}}+\frac{2}{r^{2}} \frac{\partial u_{\theta}}{\partial \theta}+\frac{1}{r^{2}} u_{r}-2 \xi \frac{\partial u_{r}}{\partial r} \\
& -\frac{\xi}{r} u_{r}-\xi^{2} u_{r}=f_{r} \quad \text { in } \mathbb{K}, \\
& -\frac{\partial^{2} u_{\theta}}{\partial r^{2}}-\frac{1}{r} \frac{\partial u_{\theta}}{\partial r}-\frac{1}{r^{2}} \frac{\partial^{2} u_{\theta}}{\partial \theta^{2}}-\frac{2}{r^{2}} \frac{\partial u_{r}}{\partial \theta}+\frac{1}{r^{2}} u_{\theta}-2 \xi \frac{\partial u_{\theta}}{\partial r} \\
& -\frac{\xi}{r} u_{\theta}-\xi^{2} u_{\theta}=f_{\theta} \quad \text { in } \mathbb{K}, \\
& \frac{\partial u_{r}}{\partial r}+\frac{1}{r} u_{r}+\frac{1}{r} \frac{\partial u_{\theta}}{\partial \theta}=0, \quad u_{r}=0 \text { on } \Gamma_{1} \cup \Gamma_{2} \\
& u_{r}=u_{\theta}=0 \quad \text { on } \Gamma_{0} \\
& \left|u_{r}(r, \theta)\right|_{r=0}<\infty, \\
& \left|u_{\theta}(r, \theta)\right|_{r=0}<\infty, \\
& u=0 \quad \text { on } \Gamma_{0} \cup \Gamma_{1} \cup \Gamma_{2} \\
& -\frac{\partial^{2} u}{\partial r^{2}}-\frac{1}{r} \frac{\partial u}{\partial r}-\frac{1}{r^{2}} \frac{\partial^{2} u}{\partial \theta^{2}}-2 \xi \frac{\partial u}{\partial r}-\frac{\xi}{r} u-\xi_{r=0}^{2} u=f
\end{aligned}
$$

where the vector field $\mathbf{f}=\left(f_{r}(r, \theta), f_{\theta}(r, \theta)\right)$ from (52) in polar coordinates is linked to the vector field $\widetilde{\mathbf{f}}=$ $\left(f_{1}\left(x_{1}, x_{2}\right), f_{2}\left(x_{1}, x_{2}\right)\right)$ in Cartesian coordinates according to relation (36) and the scalar function $f=f(r, \theta)$ from (53) is linked to the scalar function $\widetilde{f}=\widetilde{f}\left(x_{1}, x_{2}\right)$ according to relation (35). The symbol $\xi$ denotes a positive real parameter. The interest on the boundary value problems (52) and (53) is purely mathematical and there is no evidence that these problems have any practical applications. The results obtained in this subsection will be used for the analysis of the regularity properties of the solution of Maxwell's equations near polyhedral edges.

The main results concerning the regularity properties of the solution of the boundary value problems (52) and (53) are formulated in Theorems 10 and 11.

Theorem 10. Let $\tilde{f} \in L_{2}(\widetilde{\mathbb{K}}), \xi>0$, and $\lambda_{k}=k \pi / \omega, k \in$ $\mathbb{N}$, with $\omega \neq \pi$. Then the boundary value problem (53) has a unique variational solution $u=\widetilde{u} \in H_{0}^{1}(\widetilde{\mathbb{K}})$ :

(a) If $\lambda_{1}>1$, then the solution $\tilde{u} \in H^{2}(\widetilde{\mathbb{K}})$ and there exists a constant $C>0$ independent of $\widetilde{u}, \widetilde{f}$, and $\xi$ such that

$$
\|\tilde{u}\|_{H^{2}(\widetilde{\mathbb{K}})}+\xi\|\widetilde{u}\|_{H^{1}(\widetilde{\mathbb{K}})}+\xi^{2}\|\widetilde{u}\|_{L_{2}(\widetilde{\mathbb{K}})} \leq C\|\tilde{f}\|_{L_{2}(\widetilde{\mathbb{K}})}
$$

(b) If $0<\lambda_{1}<1$ and $\lambda_{2} \neq 1$, then there exists a coefficient $\gamma_{1}=\gamma_{1}(\xi)$ such that the solution $\tilde{u} \in H_{0}^{1}(\widetilde{\mathbb{K}})$ can be split as a sum of a regular and a singular part in the form

$$
\begin{array}{r}
\widetilde{u}\left(x_{1}, x_{2}\right)=u(r, \theta)=\widetilde{w}\left(x_{1}, x_{2}\right)+\gamma_{1} r^{\lambda_{1}} \mathrm{e}^{-\xi r} \sin \lambda_{1} \theta \\
\text { with } \widetilde{w} \in H^{2}(\widetilde{\mathbb{K}}) .
\end{array}
$$

The coefficient $\gamma_{1}=\gamma_{1}(\xi)$ is given explicitly by the formula

$$
\gamma_{1}(\xi):=\frac{1}{\omega \lambda_{1}} \int_{\mathbb{K}} f(r, \theta) \mathrm{e}^{\xi r} r^{1-\lambda_{k}} \sin \lambda_{k} d r d \theta .
$$

Furthermore, there exists a constant $C>0$ independent of $\widetilde{f}$ and $\xi$ such that

(i) $\xi^{1-\lambda_{1}}\left|\gamma_{1}(\xi)\right| \leq C\|\tilde{f}\|_{L_{2}(\widetilde{K})}$ as $\xi \rightarrow \infty$,

(ii) $\|\widetilde{w}\|_{H^{2}(\widetilde{\mathbb{K}})}+\xi\|\widetilde{w}\|_{H^{1}(\widetilde{\mathbb{K}})}+\xi^{2}\|\widetilde{w}\|_{L_{2}(\widetilde{\mathbb{K}})} \leq C\|\tilde{f}\|_{L_{2}(\widetilde{\mathbb{K}})}$ as $\xi \rightarrow \infty$.

For the proof of Theorem 10, see [24, pp. 171-174].

Theorem 11. Let $\widetilde{\mathbf{f}}=\left(f_{1}, f_{2}\right) \in\left(L_{2}(\widetilde{\mathbb{K}})\right)^{2}, \xi>0$, and $\lambda_{k}=$ $k \pi / \omega, k \in \mathbb{N}$, with $\omega \neq \pi$. Then there exists a unique variational solution $\widetilde{\mathbf{u}}=\left(u_{1}, u_{2}\right) \in \mathscr{H}_{0}(\mathrm{curl}, \operatorname{div}, \widetilde{\mathbb{K}})$ of the boundary value problem (52). If $\lambda_{k} \neq 2, k \in \mathbb{N}$, then there exist coefficients $\gamma_{1 k}=\gamma_{1 k}(\xi)$ such that the solution $\widetilde{\mathbf{u}}$ can be split as a sum of a regular and a singular part in the form

$$
\begin{aligned}
& \widetilde{\mathbf{u}}=\left(w_{1}, w_{2}\right)+\left(s_{r}(r, \theta), s_{\theta}(r, \theta)\right) \\
& \text { with } \widetilde{\mathbf{w}} \in\left(H^{2}(\widetilde{\mathbb{K}})\right)^{2}, \\
& \left(s_{r}(r, \theta), s_{\theta}(r, \theta)\right) \\
& =\sum_{\substack{0<\lambda_{k}<2 \\
\lambda_{k} \neq 1}} \gamma_{1 k} \mathrm{e}^{-\xi r} r^{\lambda_{k}-1}\left(\sin \lambda_{k} \theta, \cos \lambda_{k} \theta\right) .
\end{aligned}
$$

The coefficients $\gamma_{1 k}=\gamma_{1 k}(\xi)$ are given explicitly by the formula

$$
\begin{aligned}
& \gamma_{1 k}(\xi):=-\frac{1}{2 \omega\left(\lambda_{k}-1\right) R^{2 \lambda_{k}-2}} \\
& \cdot \int_{\mathbb{K}}\left(f_{r}(r, \theta) \sin \lambda_{k} \theta+f_{\theta}(r, \theta) \cos \lambda_{k} \theta\right) \\
& \cdot \mathrm{e}^{\xi r} r^{\lambda_{k}} d r d \theta .
\end{aligned}
$$

Furthermore, there exists a constant $C>0$ independent of $\tilde{\mathbf{f}}$ and $\xi$ such that

(i) $\xi^{\lambda_{k}}\left|\gamma_{1 k}(\xi)\right| \leq C\|\widetilde{\mathbf{f}}\|_{\left(L_{2}(\widetilde{\mathbb{K}})\right)^{2}}$ as $\xi \rightarrow \infty$,

(ii) $\|\widetilde{\mathbf{w}}\|_{\left(H^{2}(\widetilde{\mathbb{K}})\right)^{2}}+\xi\|\widetilde{\mathbf{w}}\|_{\left(H^{1}(\widetilde{\mathbb{K}})\right)^{2}}+\xi^{2}\|\widetilde{\mathbf{w}}\|_{\left(L_{2}(\widetilde{\mathbb{K}})\right)^{2}} \leq C\|\widetilde{\mathbf{f}}\|_{\left(L_{2}(\widetilde{\mathbb{K}})\right)^{2}}$ as $\xi \rightarrow \infty$. 
The proof of Theorem 11 is very lengthy and will be broken down into several lemmas as follows.

Lemma 12. The solution $\widetilde{\mathbf{u}}=\mathbf{u}=\left(u_{r}, u_{\theta}\right)$ of problem (52) can be represented in a Fourier series in the form

$$
\begin{aligned}
\left(u_{r}\right. & \left.(r, \theta), u_{\theta}(r, \theta)\right) \\
& =\sum_{k=1}^{\infty}\left(u_{r k}(r) \sin \lambda_{k} \theta, u_{\theta k}(r) \cos \lambda_{k} \theta\right),
\end{aligned}
$$

where the Fourier coefficients $\mathbf{u}_{k}=\left(u_{r k}, u_{\theta k}\right), k \in \mathbb{N}$, are given explicitly by the formulas

$$
\begin{aligned}
& u_{r k}(r)=-\frac{r^{\lambda_{k}-1} \mathrm{e}^{-\xi r}}{4\left(\lambda_{k}-1\right) R^{2 \lambda_{k}-2}} \\
& \cdot \int_{0}^{R} \mathrm{e}^{\xi \tau}\left(f_{r k}(\tau)+f_{\theta k}(\tau)\right) \tau^{\lambda_{k}} d \tau \\
& +\frac{r^{\lambda_{k}+1} \mathrm{e}^{-\xi r}}{4\left(\lambda_{k}+1\right) R^{2 \lambda_{k}+2}} \\
& \cdot \int_{0}^{R} \mathrm{e}^{\xi \tau}\left(-f_{r k}(\tau)+f_{\theta k}(\tau)\right) \tau^{\lambda_{k}+2} d \tau \\
& +\frac{r^{-\left(\lambda_{k}+1\right)} \mathrm{e}^{-\xi r}}{4\left(\lambda_{k}+1\right)} \int_{0}^{r} \mathrm{e}^{\xi \tau}\left(f_{r k}(\tau)-f_{\theta k}(\tau)\right) \tau^{\lambda_{k}+2} d \tau \\
& -\frac{r^{\lambda_{k}+1} \mathrm{e}^{-\xi r}}{4\left(\lambda_{k}+1\right)} \int_{r}^{R} \mathrm{e}^{\xi \tau}\left(-f_{r k}(\tau)+f_{\theta k}(\tau)\right) \tau^{-\lambda_{k}} d \tau \\
& +\frac{r^{1-\lambda_{k}} \mathrm{e}^{-\xi r}}{4\left(\lambda_{k}-1\right)} \int_{0}^{r} \mathrm{e}^{\xi \tau}\left(f_{r k}(\tau)+f_{\theta k}(\tau)\right) \tau^{\lambda_{k}} d \tau \\
& +\frac{r^{\lambda_{k}-1} \mathrm{e}^{-\xi r}}{4\left(\lambda_{k}-1\right)} \int_{r}^{R} \mathrm{e}^{\xi \tau}\left(f_{r k}(\tau)+f_{\theta k}(\tau)\right) \tau^{2-\lambda_{k}} d \tau, \\
& u_{\theta k}(r)=-\frac{r^{\lambda_{k}-1} \mathrm{e}^{-\xi r}}{4\left(\lambda_{k}-1\right) R^{2 \lambda_{k}-2}} \\
& \cdot \int_{0}^{R} e^{\xi \tau}\left(f_{r k}(\tau)+f_{\theta k}(\tau)\right) \tau^{\lambda_{k}} d \tau \\
& -\frac{r^{\lambda_{k}+1} \mathrm{e}^{-\xi r}}{4\left(\lambda_{k}+1\right) R^{2 \lambda_{k}+2}} \\
& \cdot \int_{0}^{R} \mathrm{e}^{\xi \tau}\left(-f_{r k}(\tau)+f_{\theta k}(\tau)\right) \tau^{\lambda_{k}+2} d \tau \\
& -\frac{r^{-\left(\lambda_{k}+1\right)} \mathrm{e}^{-\xi r}}{4\left(\lambda_{k}+1\right)} \int_{0}^{r} \mathrm{e}^{\xi \tau}\left(f_{r k}(\tau)-f_{\theta k}(\tau)\right) \tau^{\lambda_{k}+2} d \tau \\
& +\frac{r^{\lambda_{k}+1} \mathrm{e}^{-\xi r}}{4\left(\lambda_{k}+1\right)} \int_{r}^{R} \mathrm{e}^{\xi \tau}\left(-f_{r k}(\tau)+f_{\theta k}(\tau)\right) \tau^{-\lambda_{k}} d \tau
\end{aligned}
$$

$$
\begin{aligned}
& +\frac{r^{1-\lambda_{k}} \mathrm{e}^{-\xi r}}{4\left(\lambda_{k}-1\right)} \int_{0}^{r} \mathrm{e}^{\xi \tau}\left(f_{r k}(\tau)+f_{\theta k}(\tau)\right) \tau^{\lambda_{k}} d \tau \\
& +\frac{r^{\lambda_{k}-1} \mathrm{e}^{-\xi r}}{4\left(\lambda_{k}-1\right)} \int_{r}^{R} \mathrm{e}^{\xi \tau}\left(f_{r k}(\tau)+f_{\theta k}(\tau)\right) \tau^{2-\lambda_{k}} d \tau .
\end{aligned}
$$

Lemma 13. Let

$$
\begin{aligned}
\gamma_{1 k}(\xi)= & -\frac{1}{4\left(\lambda_{k}-1\right) R^{2 \lambda_{k}-2}} \\
& \cdot \int_{0}^{R} \mathrm{e}^{\xi \tau}\left(f_{r k}(\tau)+f_{\theta k}(\tau)\right) \tau^{\lambda_{k}} d \tau .
\end{aligned}
$$

If $0<\lambda_{k}<2$, then there exists a constant $C>0$ independent of $\mathbf{f}_{k}$ and $\xi$ such that

$$
\left|\gamma_{1 k}(\xi)\right| \leq C \xi^{-\lambda_{k}}\left\|\mathbf{f}_{k}\right\|_{\left(L_{2,1 / 2}(0, R)\right)^{2}}
$$

Proof. Application of Cauchy-Schwartz inequality and the substitution $\xi \tau=s$ lead to the estimates

$$
\begin{aligned}
& \left|\gamma_{1 k}\right|^{2} \leq C\left(\int_{0}^{R} \mathrm{e}^{\xi \tau}\left(f_{r k}(\tau)+f_{\theta k}(\tau)\right) \tau^{\lambda_{k}} d \tau\right)^{2} \\
& \leq C \int_{0}^{R}\left(\left|f_{r k}(\tau)\right|^{2}+\left|f_{\theta k}(\tau)\right|^{2}\right) \tau d \tau \int_{0}^{R} \mathrm{e}^{2 \xi \tau} \tau^{2 \lambda_{k}-1} d \tau \\
& \leq C \xi^{-2 \lambda_{k}}\left\|f_{k}\right\|_{L_{2,1 / 2}(0, R)^{2}}^{2} \int_{0}^{\widetilde{R}} \mathrm{e}^{2 s} s^{2 \lambda_{k}-1} d s \\
& \leq C \xi^{-2 \lambda_{k}}\left\|\mathbf{f}_{k}\right\|_{L_{2,1 / 2}(0, R)^{2}}^{2} .
\end{aligned}
$$

Lemma 14. Let

$$
T_{k}(r)=r^{\lambda_{k}-1} \mathrm{e}^{-\xi r}
$$

If $\lambda_{k}>2$, then there exists a constant $C>0$ independent of $\xi$ such that

$$
t_{1}:=\int_{0}^{R}\left|T_{k}(\tau)\right|^{2} \tau d \tau \leq C \xi^{-2 \lambda_{k}} \quad \text { as } \xi \longrightarrow \infty,
$$

(ii)

$$
t_{2}:=\int_{0}^{R}\left\{\left|T_{k}^{\prime}(\tau)\right|^{2}+\lambda_{k}^{2}\left|\frac{T_{k}(\tau)}{\tau}\right|^{2}\right\} \tau d \tau \leq C \xi^{2-2 \lambda_{k}}
$$


(iii)

$$
\begin{aligned}
t_{3} & :=\int_{0}^{R}\left\{\left|T_{k}^{\prime \prime}(\tau)\right|^{2}+2 \lambda_{k}^{2}\left|\frac{T_{k}^{\prime}(\tau)}{\tau}-\frac{T_{k}(\tau)}{\tau^{2}}\right|^{2}\right. \\
& \left.+\left|\frac{T_{k}^{\prime}(\tau)}{\tau}-\frac{\lambda_{k}^{2}}{\tau^{2}} T_{k}(\tau)\right|^{2}\right\} \tau d \tau \leq C \xi^{4-2 \lambda_{k}}
\end{aligned}
$$

$$
\text { as } \xi \longrightarrow \infty \text {. }
$$

Proof. Using the substitution $\xi \tau=s$ one easily verifies the estimates

$$
\begin{aligned}
t_{1} & =\int_{0}^{R} \mathrm{e}^{-2 \xi \tau} \tau^{2 \lambda_{k}-1} d \tau \leq C \xi^{-2 \lambda_{k}} \int_{0}^{\widetilde{R}} \mathrm{e}^{-2 s} s^{2 \lambda_{k}-1} d s \\
& \leq C \xi^{-2 \lambda_{k}}, \\
t_{2} & \leq C\left(\xi^{2} \int_{0}^{R} \mathrm{e}^{-2 \xi \tau} \tau^{2 \lambda_{k}-1} d \tau+\int_{0}^{R} \mathrm{e}^{-2 \xi \tau} \tau^{2 \lambda_{k}-3} d \tau\right) \\
& \leq C \xi^{2-2 \lambda_{k}}\left(\int_{0}^{\widetilde{R}} \mathrm{e}^{-2 s} s^{2 \lambda_{k}-1} d s+\int_{0}^{\widetilde{R}} \mathrm{e}^{-2 s} s^{2 \lambda_{k}-3} d s\right) \\
& \leq C \xi^{2-2 \lambda_{k}}, \\
t_{3} & \leq C\left(\xi^{4} \int_{0}^{R} \mathrm{e}^{-2 \xi \tau} \tau^{2 \lambda_{k}-1} d \tau+\xi^{2} \int_{0}^{R} \mathrm{e}^{-2 \xi \tau} \tau^{2 \lambda_{k}-3} d \tau\right. \\
& \left.+\int_{0}^{R} \mathrm{e}^{-2 \xi \tau} \tau^{2 \lambda_{k}-5} d \tau\right) \leq C \xi^{4-2 \lambda_{k}}\left(\int_{0}^{\widetilde{R}} \mathrm{e}^{-2 s} s^{2 \lambda_{k}-1} d s\right. \\
& \left.+\int_{0}^{\widetilde{R}} \mathrm{e}^{-2 s} s^{2 \lambda_{k}-3} d s+\int_{0}^{\widetilde{R}} \mathrm{e}^{-2 s} s^{2 \lambda_{k}-5} d s\right) \leq C \xi^{4-2 \lambda_{k}} .
\end{aligned}
$$

The following lemmas can be proved by analogy. We omit the proofs for the sake of brevity.

Lemma 15. Let

$$
\begin{aligned}
\delta_{k}= & \frac{1}{4\left(\lambda_{k}+1\right) R^{2 \lambda_{k}+2}} \\
& \cdot \int_{0}^{R} \mathrm{e}^{\xi \tau}\left(-f_{r k}(\tau)+f_{\theta k}(\tau)\right) \tau^{\lambda_{k}+2} d \tau .
\end{aligned}
$$

Then there exists a constant $C>0$ independent of $\mathbf{f}_{k}$ and $\xi$ such that

$$
\left|\delta_{k}(\xi)\right| \leq C \xi^{-\left(\lambda_{k}+2\right)}\left\|\mathbf{f}_{k}\right\|_{L_{2,1 / 2}(0, R)^{2}} .
$$

Lemma 16. Let

$$
F_{k}(r)=r^{\lambda_{k}+1} \mathrm{e}^{-\xi r}
$$

Then there exists a constant $C>0$ independent of $\xi$ such that

$$
s_{1}:=\int_{0}^{R}\left|F_{k}(\tau)\right|^{2} \tau d \tau \leq C \xi^{-2\left(\lambda_{k}+2\right)} \quad \text { as } \xi \longrightarrow \infty,
$$

(ii)

$$
s_{2}:=\int_{0}^{R}\left\{\left|F_{k}^{\prime}(\tau)\right|^{2}+\lambda_{k}^{2}\left|\frac{F_{k}(\tau)}{\tau}\right|^{2}\right\} \tau d \tau \leq C \xi^{-2\left(\lambda_{k}+1\right)}
$$

as $\xi \longrightarrow \infty$,

(iii)

$$
\begin{aligned}
s_{3} & :=\int_{0}^{R}\left\{\left|F_{k}^{\prime \prime}(\tau)\right|^{2}+2 \lambda_{k}^{2}\left|\frac{F_{k}^{\prime}(\tau)}{\tau}-\frac{F_{k}(\tau)}{\tau^{2}}\right|^{2}\right. \\
& \left.+\left|\frac{F_{k}^{\prime}(\tau)}{\tau}-\frac{\lambda_{k}^{2}}{\tau^{2}} F_{k}(\tau)\right|^{2}\right\} \tau d \tau \leq C \xi^{-2 \lambda_{k}}
\end{aligned}
$$

as $\xi \longrightarrow \infty$.

\section{Lemma 17. Let}

$$
\begin{aligned}
& G_{k}(r ; \xi) \\
& =\frac{r^{-\left(\lambda_{k}+1\right)} \mathrm{e}^{-\xi r}}{4\left(\lambda_{k}+1\right)} \int_{0}^{r} \mathrm{e}^{\xi \tau}\left(f_{r k}(\tau)-f_{\theta k}(\tau)\right) \tau^{\lambda_{k}+2} d \tau \\
& \quad-\frac{r^{\left(\lambda_{k}+1\right)} \mathrm{e}^{-\xi r}}{4\left(\lambda_{k}+1\right)} \int_{r}^{R} \mathrm{e}^{\xi \tau}\left(-f_{r k}(\tau)+f_{\theta k}(\tau)\right) \tau^{-\lambda_{k}} d \tau .
\end{aligned}
$$

Then there exists a constant $C>0$ independent of $\mathbf{f}_{k}$ and $\xi$ such that

(i)

$$
g_{1}:=\int_{0}^{R}\left|G_{k}(\tau)\right|^{2} \tau d \tau \leq C \xi^{-4}\left\|\mathbf{f}_{k}\right\|_{L_{2,1 / 2}(0, R)}^{2}
$$

as $\xi \longrightarrow \infty$,

(ii)

$$
\begin{aligned}
g_{2} & :=\int_{0}^{R}\left\{\left|G_{k}^{\prime}(\tau)\right|^{2}+\lambda_{k}^{2}\left|\frac{G_{k}(\tau)}{\tau}\right|^{2}\right\} \tau d \tau \\
& \leq C \xi^{-2}\left\|\mathbf{f}_{k}\right\|_{L_{2,1 / 2}(0, R)}^{2} \quad \text { as } \xi \longrightarrow \infty,
\end{aligned}
$$

(iii)

$$
\begin{aligned}
g_{3} & :=\int_{0}^{R}\left\{\left|G_{k}^{\prime \prime}(\tau)\right|^{2}+2 \lambda_{k}^{2}\left|\frac{G_{k}^{\prime}(\tau)}{\tau}-\frac{G_{k}(\tau)}{\tau^{2}}\right|^{2}\right. \\
& \left.+\left|\frac{G_{k}^{\prime}(\tau)}{\tau}-\frac{\lambda_{k}^{2}}{\tau^{2}} G_{k}(\tau)\right|^{2}\right\} \tau d \tau \leq C\left\|\mathbf{f}_{k}\right\|_{L_{2,1 / 2}(0, R)}^{2}
\end{aligned}
$$


Lemma 18. Let

$$
\begin{aligned}
& I_{k}(r) \\
& \quad=\frac{r^{1-\lambda_{k}} \mathrm{e}^{-\xi r}}{4\left(\lambda_{k}-1\right)} \int_{0}^{r} \mathrm{e}^{\xi \tau}\left(f_{r k}(\tau)+f_{\theta k}(\tau)\right) \tau^{\lambda_{k}} d \tau \\
& \quad+\frac{r^{\lambda_{k}-1} \mathrm{e}^{-\xi r}}{4\left(\lambda_{k}-1\right)} \int_{r}^{R} \mathrm{e}^{\xi \tau}\left(f_{r k}(\tau)+f_{\theta k}(\tau)\right) \tau^{2-\lambda_{k}} d \tau .
\end{aligned}
$$

Then there exists a constant $C>0$ independent of $\mathbf{f}_{k}$ and $\xi$ such that

(i)

$$
i_{1}:=\int_{0}^{R}\left|I_{k}(\tau)\right|^{2} \tau d \tau \leq C \xi^{-4}\left\|\mathbf{f}_{k}\right\|_{L_{2,1 / 2}(0, R)}^{2}
$$

$$
\text { as } \xi \longrightarrow \infty \text {, }
$$

(ii)

$$
\begin{aligned}
i_{2} & :=\int_{0}^{R}\left\{\left|I_{k}^{\prime}(\tau)\right|^{2}+\lambda_{k}^{2}\left|\frac{I_{k}(\tau)}{\tau}\right|^{2}\right\} \tau d \tau \\
& \leq C \xi^{-2}\left\|\mathbf{f}_{k}\right\|_{L_{2,1 / 2}(0, R)}^{2} \text { as } \xi \longrightarrow \infty,
\end{aligned}
$$

(iii)

$$
\begin{aligned}
i_{3} & :=\int_{0}^{R}\left\{\left|I_{k}^{\prime \prime}(\tau)\right|^{2}+2 \lambda_{k}^{2}\left|\frac{I_{k}^{\prime}(\tau)}{\tau}-\frac{I_{k}(\tau)}{\tau^{2}}\right|^{2}\right. \\
& \left.+\left|\frac{I_{k}^{\prime}(\tau)}{\tau}-\frac{\lambda_{k}^{2}}{\tau^{2}} I_{k}(\tau)\right|^{2}\right\} \tau d \tau \leq C\left\|\mathbf{f}_{k}\right\|_{L_{2,1 / 2}(0, R)}^{2}
\end{aligned}
$$

$$
\text { as } \xi \longrightarrow \infty \text {. }
$$
follows.

The proof of Theorem 11 can now be summarised as

Proof (Theorem 11). The results of Theorem 11 follow by combining Lemmas 12-18, taking note of the definition of the representation of norms of functions as series of norms of their Fourier coefficients by means of generalized Parseval identities; see, for example, $[13,18]$.

\section{Maxwell's Equations in Domains with Polyhedral Edges}

In this section, we consider and analyze the Maxwell equations (25) in three-dimensional domains with polyhedral edges and prove Theorem 6.

5.1. Maxwell's Equations in a Three-Dimensional Wedge. We consider first a three-dimensional domain of the form $\Omega=$ $\widetilde{\mathbb{K}} \times(0, l)$, where $l>0$ is a real constant and $\widetilde{\mathbb{K}} \subset \mathbb{R}^{2}$; see

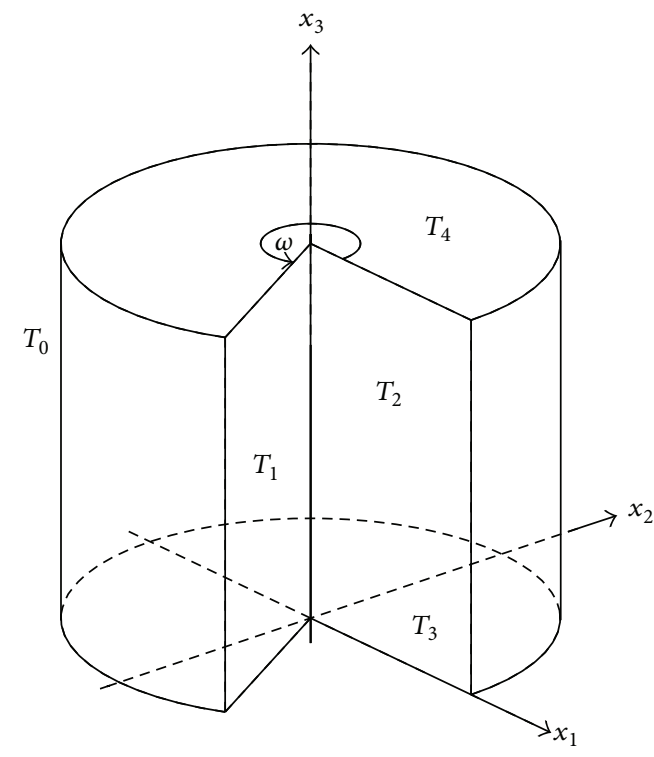

FIgURE 2: A three-dimensional wedge.

Figure 1. We will use the same notations as in Section 4.1 for $\widetilde{\mathbb{K}}$. Thus the boundary $\Gamma$ of $\Omega$ can be represented in the form $\Gamma=\bar{T}_{0} \cup \bar{T}_{1} \cup \bar{T}_{2} \cup \bar{T}_{3} \cup \bar{T}_{4}$, where $T_{3}=\Omega \times\{0\}, T_{4}=\Omega \times\{l\}$, and $T_{j}=\Gamma_{j} \times(0, l), j=0,1,2$; see Figure 2 .

For a given vector field $\mathbf{f} \in\left(L_{2}(\Omega)\right)^{3}$, let $\mathbf{u} \in \mathscr{H}_{0}$ (curl, $\operatorname{div}, \Omega$ ) be the variational solution of the boundary value problem

$$
\begin{aligned}
& \text { curl curl } \mathbf{u}-\operatorname{grad} \operatorname{div} \mathbf{u}=\mathbf{f} \quad \text { in } \Omega \text {, } \\
& \mathbf{u} \wedge \mathbf{n}=\mathbf{0} \quad \text { on } T_{1} \cup T_{2} \cup T_{3} \cup T_{4} \text {, } \\
& \operatorname{div} \mathbf{u}=0 \quad \text { on } T_{1} \cup T_{2} \cup T_{3} \cup T_{4}, \\
& \mathbf{u}=\mathbf{0} \text { on } T_{0} .
\end{aligned}
$$

We observe that the systems of trigonometric functions $\left\{\sin \left(n \pi x_{3} / l\right): n \in \mathbb{N}\right\}$ and $\left\{\cos \left(n \pi x_{3} / l\right): n \in \mathbb{N}_{0}\right\}$ $\left(\mathbb{N}_{0}:=\{0,1,2, \ldots\}\right)$ are orthogonal and complete in $L_{2}(0, l)$; see $[25,26]$. Thus functions $\mathbf{v} \in\left(L_{2}(\Omega)\right)^{3}$ can be characterized by their Fourier coefficients as follows.

Lemma 19. (1) Let $\mathbf{v}=\left(v_{1}, v_{2}, v_{3}\right) \in\left(L_{2}(\Omega)\right)^{3}$. Then there exist in $\left(L_{2}(\widetilde{\mathbb{K}})\right)^{3}$ Fourier coefficients $\left\{\mathbf{v}_{n}=\left(v_{1 n}, v_{2 n}, v_{3 n}\right): n \in \mathbb{N}_{0}\right\}$ of $\mathbf{v}$ defined by

$$
\begin{aligned}
& v_{\text {in }}\left(x_{1}, x_{2}\right)=\frac{2}{l} \int_{0}^{l} v_{i}\left(x_{1}, x_{2}, x_{3}\right) \sin \frac{n \pi x_{3}}{l}, \quad i=1,2, \\
& v_{3 n}\left(x_{1}, x_{2}\right)=\frac{2}{l} \int_{0}^{l} v_{3}\left(x_{1}, x_{2}, x_{3}\right) \cos \frac{n \pi x_{3}}{l}
\end{aligned}
$$


and satisfying the relations

$$
\begin{aligned}
v_{1}\left(x_{1}, x_{2}, x_{3}\right)= & \sum_{n=1}^{\infty} v_{1 n}\left(x_{1}, x_{2}\right) \sin \frac{n \pi x_{3}}{l}, \\
v_{2}\left(x_{1}, x_{2}, x_{3}\right)= & \sum_{n=1}^{\infty} v_{2 n}\left(x_{1}, x_{2}\right) \sin \frac{n \pi x_{3}}{l}, \\
v_{3}\left(x_{1}, x_{2}, x_{3}\right)= & \frac{1}{2} v_{30}\left(x_{1}, x_{2}\right) \\
& +\sum_{n=1}^{\infty} v_{3 n}\left(x_{1}, x_{2}\right) \cos \frac{n \pi x_{3}}{l} .
\end{aligned}
$$

Moreover, Parseval's identity holds in the form

$$
\begin{array}{r}
\left\|v_{i}\right\|_{L_{2}(\Omega)}^{2}=\frac{l}{4}\left\|v_{i 0}\right\|_{L_{2}(\widetilde{\mathbb{K}})}^{2}+\frac{l}{2} \sum_{n=1}^{\infty}\left\|v_{i n}\right\|_{L_{2}(\widetilde{\mathbb{K}})}^{2}<\infty, \\
i=1,2,3 .
\end{array}
$$

(2) For $\mathbf{v} \in \mathscr{H}_{0}$ (curl, div, $\Omega$ ), relations (84), (85), and (86) hold and additionally

$$
\begin{aligned}
& \|\mathbf{v}\|_{\mathscr{H}_{0}(\mathrm{curl}, \mathrm{div}, \Omega)}^{2}=\frac{l}{4}\left\|v_{30}\right\|_{H^{1}(\widetilde{\mathbb{K}})}^{2}+\frac{l}{2} \sum_{n=1}^{\infty}\left\{\left\|\mathbf{v}_{n}\right\|_{\left(L_{2}(\widetilde{\mathbb{K}})\right)^{3}}^{2}\right. \\
& +\left\|\frac{\partial v_{2 n}}{\partial x_{1}}-\frac{\partial v_{1 n}}{\partial x_{2}}\right\|_{L_{2}(\widetilde{\mathbb{K}})}^{2} \\
& +\left\|\frac{\partial v_{1 n}}{\partial x_{1}}+\frac{\partial v_{2 n}}{\partial x_{2}}-\frac{n \pi}{l} v_{3 n}\right\|_{L_{2}(\widetilde{\mathbb{K}})}^{2} \\
& \left.+\left\|\frac{n \pi}{l} v_{1 n}-\frac{\partial v_{3 n}}{\partial x_{1}}\right\|_{L_{2}(\widetilde{\mathbb{K}})}^{2}+\left\|\frac{\partial v_{3 n}}{\partial x_{2}}-\frac{n \pi}{l} u_{2 n}\right\|_{L_{2}(\widetilde{\mathbb{K}})}^{2}\right\} \\
& \quad<\infty .
\end{aligned}
$$

For the study of the Fourier coefficients $\left\{\mathbf{u}_{n}=\right.$ $\left.\left(u_{1 n}, u_{2 n}, u_{3 n}\right): n \in \mathbb{N}_{0}\right\}$ of the solution $\mathbf{u} \in \mathscr{H}_{0}(\operatorname{curl}, \operatorname{div}, \Omega)$ of the boundary value problem (83), we introduce on $\widetilde{\mathbb{K}}$ the spaces

$$
\begin{gathered}
\mathscr{Y}(\widetilde{\mathbb{K}}):=\left\{\mathbf{w}=\left(w_{1}, w_{2}, w_{3}\right) \in\left(L_{2}(\widetilde{\mathbb{K}})\right)^{3}: \frac{\partial w_{2}}{\partial x_{1}}\right. \\
\left.-\frac{\partial w_{1}}{\partial x_{2}}, \frac{\partial w_{1}}{\partial x_{1}}+\frac{\partial w_{2}}{\partial x_{2}}, \frac{\partial w_{3}}{\partial x_{1}}, \frac{\partial w_{3}}{\partial x_{2}} \in L_{2}(\widetilde{\mathbb{K}})\right\}, \\
\mathscr{Y}_{0}(\widetilde{\mathbb{K}}):=\left\{w=\left(w_{1}, w_{2}, w_{3}\right) \in \mathcal{Y}(\widetilde{\mathbb{K}}): w_{1} n_{2}\right. \\
-w_{2} n_{1}=0, \frac{\partial w_{1}}{\partial x_{1}}+\frac{\partial w_{2}}{\partial x_{2}}=0 \text { on } \Gamma_{1} \cup \Gamma_{2}, w_{3} \\
\left.=0 \text { on } \Gamma_{1} \cup \Gamma_{2}, w_{1}=w_{2}=w_{3}=0 \text { on } \Gamma_{0}\right\},
\end{gathered}
$$

where $\mathbf{n}=\left(n_{1}, n_{2}\right)$ denotes the unit outward normal on the boundary $\Gamma_{1} \cup \Gamma_{2}$. These spaces are equipped with the norm

$$
\begin{gathered}
\|\mathbf{w}\|_{\mathscr{Y}(\widetilde{\mathbb{K}})}=\left(\|\mathbf{w}\|_{\left(L_{2}(\widetilde{\mathbb{K}})\right)^{3}}^{2}+\left\|\frac{\partial w_{2}}{\partial x_{1}}-\frac{\partial w_{1}}{\partial x_{2}}\right\|_{L_{2}(\widetilde{\mathbb{K}})}^{2}\right. \\
\left.+\left\|\frac{\partial w_{1}}{\partial x_{1}}+\frac{\partial w_{2}}{\partial x_{2}}\right\|_{L_{2}(\widetilde{\mathbb{K}})}^{2}+\left|w_{3}\right|_{H^{1}(\widetilde{\mathbb{K}})}^{2}\right)^{1 / 2} .
\end{gathered}
$$

Clearly, the spaces $\mathscr{Y}(\widetilde{\mathbb{K}})$ and $\mathscr{Y}_{0}(\widetilde{\mathbb{K}})$ endowed with the norm (89) are Hilbert spaces. Indeed, we observe the identities

$$
\begin{aligned}
& \mathscr{Y}(\widetilde{\mathbb{K}})=\mathscr{H}(\text { curl, div }, \widetilde{\mathbb{K}}) \times H^{1}(\widetilde{\mathbb{K}}), \\
& \mathscr{Y}_{0}(\widetilde{\mathbb{K}})=\mathscr{H}_{0}(\text { curl, div }, \widetilde{\mathbb{K}}) \times H_{0}^{1}(\widetilde{\mathbb{K}})
\end{aligned}
$$

Lemma 20. Let $\left\{\mathbf{v}_{n}=\left(v_{1 n}, v_{2 n}, v_{3 n}\right): n \in \mathbb{N}_{0}\right\}$ denote the Fourier coefficients of a function $\mathbf{v} \in \mathscr{H}_{0}$ (curl, div, $\Omega$ ) defined according to relation (84). Then $\mathbf{v}_{n} \in \mathscr{Y}_{0}(\widetilde{\mathbb{K}})$ for any $n \in \mathbb{N}_{0}$.

Proof. It follows from (84) that $\mathbf{v}_{0}=\left(0,0, v_{30}\right)$. The completeness relationship (87) infers then that $v_{30} \in H^{1}(\widetilde{\mathbb{K}})$ and consequently $\mathbf{v}_{0} \in \mathcal{Y}(\widetilde{\mathbb{K}})$. Further, with the help of the triangle inequality and relation (87) we get the estimates

$$
\begin{aligned}
& \left\|\frac{\partial v_{3 n}}{\partial x_{1}}\right\|_{L_{2}(\widetilde{\mathbb{K}})} \\
& \quad \leq\left(\left\|\frac{n \pi}{l} v_{1 n}-\frac{\partial v_{3 n}}{\partial x_{1}}\right\|_{L_{2}(\widetilde{\mathbb{K}})}+\frac{n \pi}{l}\left\|v_{1 n}\right\|_{L_{2}(\widetilde{\mathbb{K}})}\right)<\infty, \\
& \left\|\frac{\partial v_{3 n}}{\partial x_{2}}\right\|_{L_{2}(\widetilde{\mathbb{K}})} \\
& \quad \leq\left(\left\|\frac{\partial v_{3 n}}{\partial x_{2}}-\frac{n \pi}{l} v_{2 n}\right\|_{L_{2}(\widetilde{\mathbb{K}})}+\frac{n \pi}{l}\left\|v_{2 n}\right\|_{L_{2}(\widetilde{\mathbb{K}})}\right)<\infty, \\
& \left\|\frac{\partial v_{1 n}}{\partial x_{1}}+\frac{\partial v_{2 n}}{\partial x_{2}}\right\|_{L_{2}(\widetilde{\mathbb{K}})} \\
& \quad \leq\left(\left\|\frac{\partial v_{1 n}}{\partial x_{1}}+\frac{\partial v_{2 n}}{\partial x_{2}}+\frac{n \pi}{l} v_{3 n}\right\|_{L_{2}(\widetilde{\mathbb{K}})}+\frac{n \pi}{l}\left\|v_{3 n}\right\|_{L_{2}(\widetilde{\mathbb{K}})}\right) \\
& \quad<\infty .
\end{aligned}
$$

Hence, taking into account the definition of $\mathscr{Y}(\widetilde{\mathbb{K}})$ and the completeness relationship (87) we get $\mathbf{v}_{n} \in \mathcal{Y}(\widetilde{\mathbb{K}}), n \in \mathbb{N}_{0}$. The boundary conditions follow from the boundary conditions in $\mathscr{H}_{0}(\operatorname{curl}, \operatorname{div}, \Omega$ ).

Lemma 21. For $\mathbf{f} \in\left(L_{2}(\Omega)\right)^{3}$, let $\mathbf{u} \in \mathscr{H}_{0}(\operatorname{curl}, \operatorname{div}, \Omega)$ be the solution of the boundary value problem (83). Let $\left\{\mathbf{u}_{n}=\right.$ $\left.\left(u_{1 n}, u_{2 n}, u_{3 n}\right)=:\left(\widetilde{\mathbf{u}}_{n}, u_{3 n}\right): n \in \mathbb{N}_{0}\right\}$ and $\left\{\mathbf{f}_{n}=\left(f_{1 n}, f_{2 n}\right.\right.$, $\left.\left.f_{3 n}\right)=:\left(\widetilde{\mathbf{f}}_{n}, f_{3 n}\right): n \in \mathbb{N}_{0}\right\}$ denote the Fourier coefficients 
of $\mathbf{u}$ and $\mathbf{f}$, respectively, defined according to (84). Then the functions $\mathbf{u}_{n}=\left(\widetilde{\mathbf{u}}_{n}, u_{3 n}\right), n \in \mathbb{N}_{0}$, satisfy the relations $\widetilde{\mathbf{u}}_{n} \in$ $\mathscr{H}_{0}(\mathrm{curl}, \operatorname{div}, \widetilde{\mathbb{K}})$ and $u_{3 n} \in H_{0}^{1}(\widetilde{\mathbb{K}})$ and are the solutions of the boundary value problems

curl curl $\widetilde{\mathbf{u}}_{n}-\operatorname{grad} \operatorname{div} \widetilde{\mathbf{u}}_{n}+\left(\frac{n \pi}{l}\right)^{2} \widetilde{\mathbf{u}}_{n}=\widetilde{\mathbf{f}}_{n} \quad$ in $\widetilde{\mathbb{K}}$,

$$
\begin{aligned}
u_{1 n} n_{2}-u_{2 n} n_{1} & =0 \\
\operatorname{div} \widetilde{\mathbf{u}}_{n} & =0 \\
& \text { on } \Gamma_{1} \cup \Gamma_{2}, \\
\widetilde{\mathbf{u}}_{n} & =0 \quad \text { on } \Gamma_{0}, \\
-\Delta u_{3 n}+\left(\frac{n \pi}{l}\right)^{2} u_{3 n} & =f_{3 n} \quad \text { in } \widetilde{\mathbb{K}} \\
u_{3 n} & =0 \quad \text { on } \partial \widetilde{\mathbb{K}} .
\end{aligned}
$$

Proof. Problems (92) are obtained directly from (83) by substituting the functions $\mathbf{u}$ and $\mathbf{f}$ by their respective Fourier series defined according to (85), differentiating term by term and comparing coefficients. The assertions $\widetilde{\mathbf{u}}_{n} \in \mathscr{H}_{0}($ curl, div, $\widetilde{\mathbb{K}})$ and $u_{3 n} \in H_{0}^{1}(\widetilde{\mathbb{K}})$ follow from Lemma 20.

We observe that in local polar coordinates $r$ and $\theta$ problems (92) take the form

$$
\begin{aligned}
& -\frac{\partial^{2} u_{r n}}{\partial r^{2}}-\frac{1}{r} \frac{\partial u_{r n}}{\partial r}-\frac{1}{r^{2}} \frac{\partial^{2} u_{r n}}{\partial \theta^{2}}+\frac{2}{r^{2}} \frac{\partial u_{\theta n}}{\partial \theta}+\frac{1}{r^{2}} u_{r n} \\
& +\frac{n^{2} \pi^{2}}{l^{2}} u_{r n}=f_{r n} \text { in } \mathbb{K}, \\
& -\frac{\partial^{2} u_{\theta n}}{\partial r^{2}}-\frac{1}{r} \frac{\partial u_{\theta n}}{\partial r}-\frac{1}{r^{2}} \frac{\partial^{2} u_{\theta n}}{\partial \theta^{2}}-\frac{2}{r^{2}} \frac{\partial u_{r n}}{\partial \theta}+\frac{1}{r^{2}} u_{\theta n} \\
& \quad+\frac{n^{2} \pi^{2}}{l^{2}} u_{\theta n}=f_{\theta n} \text { in } \mathbb{K}, \\
& \frac{\partial u_{r n}}{\partial r}+\frac{1}{r} u_{r n}+\frac{1}{r} \frac{\partial u_{\theta n}}{\partial \theta}=0, \quad u_{r n}=0 \text { on } \Gamma_{1} \cup \Gamma_{2}, \\
& \left|u_{r n}(r, \theta)\right|_{r=0}<\infty, \\
& \left|u_{\theta n}(r, \theta)\right|_{r=0}<\infty, \\
& u_{r n}(R, \theta)=u_{\theta n}(R, \theta)=0, \\
& -\frac{\partial^{2} u_{3 n}}{\partial r^{2}}-\frac{1}{r} \frac{\partial u_{3 n}}{\partial r}-\frac{1}{r^{2}} \frac{\partial^{2} u_{3 n}}{\partial \theta^{2}}+\frac{n^{2} \pi^{2}}{l^{2}} u_{3 n}=f_{3 n}
\end{aligned}
$$

$$
\begin{aligned}
& \left.u_{3 n}(r, \theta)\right|_{\theta=0}=0, \\
& \left.u_{3 n}(r, \theta)\right|_{\theta=\omega}=0 .
\end{aligned}
$$

We will need the following notations:

$$
\begin{aligned}
& f_{r n}^{*}=f_{r n}-2 \frac{n \pi}{l} \frac{\partial u_{r n}}{\partial r}-\frac{n \pi}{l} \frac{u_{r n}}{r}-2 \frac{n^{2} \pi^{2}}{l^{2}} u_{r n}, \\
& f_{\theta n}^{*}=f_{\theta n}-2 \frac{n \pi}{l} \frac{\partial u_{\theta n}}{\partial r}-\frac{n \pi}{l} \frac{u_{\theta n}}{r}-2 \frac{n^{2} \pi^{2}}{l^{2}} u_{\theta n}, \\
& f_{3 n}^{*}=f_{3 n}-2 \frac{n \pi}{l} \frac{\partial u_{3 n}}{\partial r}-\frac{n \pi}{l} \frac{u_{3 n}}{r}-2 \frac{n^{2} \pi^{2}}{l^{2}} u_{3 n},
\end{aligned}
$$

where $\mathbf{f}_{n}=\left(f_{r n}, f_{\theta n}, f_{3 n}\right)$ and $\mathbf{u}_{n}=\left(u_{r n}, u_{\theta n}, u_{3 n}\right), n \in \mathbb{N}_{0}$, are taken from (93). Obviously $\mathbf{f}_{n}^{*}=\left(f_{r n}^{*}, f_{\theta n}^{*}, f_{3 n}^{*}\right) \in\left(L_{2}(\widetilde{\mathbb{K}})\right)^{3}$.

Theorem 22. Let $\widetilde{\mathbb{K}}$ be a circular sector with angle $\omega \in(0,2 \pi)$, $\omega \neq \pi$, and let $\lambda_{k}:=k \pi / \omega, k \in \mathbb{N}$. For each $\mathbf{f}_{n}=$ $\left(f_{1 n}, f_{2 n}, f_{3 n}\right) \in\left(L_{2}(\widetilde{\mathbb{K}})\right)^{3}$, let $\mathbf{u}_{n}=\left(u_{1 n}, u_{2 n}, u_{3 n}\right) \in \mathcal{Y}_{0}(\widetilde{\mathbb{K}})$, $n \in \mathbb{N}_{0}$, be the solutions of the boundary value problems (92). If $\lambda_{k} \neq 2, k \in \mathbb{N}$, then there exist coefficients $\gamma_{n k}$ and $\gamma_{n}$ such that the solutions $\mathbf{u}_{n}, n \in \mathbb{N}_{0}$, can be represented in the form

$$
\begin{gathered}
\mathbf{u}_{n}=\left(w_{1 n}, w_{2 n}, w_{3 n}\right)+\left(s_{r n}, s_{\theta n}, s_{3 n}\right) \\
\text { with } \mathbf{w}_{n} \in\left(L_{2}(\widetilde{\mathbb{K}})\right)^{3}, \\
s_{r n}(r, \theta):=\sum_{\substack{0<\lambda_{k}<2 \\
\lambda_{k} \neq 1}} \gamma_{n k} \mathrm{e}^{-(n \pi / l) r} r^{\lambda_{k}-1} \sin \lambda_{k} \theta, \\
s_{\theta n}(r, \theta):=\sum_{\substack{0<\lambda_{k}<2 \\
\lambda_{k} \neq 1}} \gamma_{n k} \mathrm{e}^{-(n \pi / l) r} r^{\lambda_{k}-1} \cos \lambda_{k} \theta, \\
s_{3 n}(r, \theta):=\gamma_{n} \mathrm{e}^{-(n \pi / l) r} r^{\lambda_{1}} \sin \lambda_{1} \theta \quad \text { if } 0<\lambda_{1}<1 .
\end{gathered}
$$

The coefficients $\gamma_{n k}$ and $\gamma_{n}$ of the expansion (95) are given explicitly by the formulas

$$
\begin{aligned}
& \gamma_{n k}:=-\frac{R^{2-2 \lambda_{k}}}{2 \omega\left(\lambda_{k}-1\right)} \\
& \cdot \int_{\mathbb{K}} \mathrm{e}^{(n \pi / l) r}\left(f_{r n}^{*}(r, \theta) \sin \lambda_{k} \theta+f_{\theta n}^{*}(r, \theta) \cos \lambda_{k} \theta\right)
\end{aligned}
$$

$$
\cdot r^{\lambda_{k}} d r d \theta
$$$$
\gamma_{n}:=\frac{1}{\omega \lambda_{1}} \int_{\mathbb{K}} \mathrm{e}^{(n \pi / l) r} f_{3 n}^{*}(r, \theta) \sin \left(\lambda_{1} \theta\right) r^{1-\lambda_{1}} d r d \theta,
$$ 
where the functions $\mathbf{f}_{n}^{*}=\left(f_{r n}^{*}, f_{\theta n}^{*}, f_{3 n}^{*}\right), n \in \mathbb{N}_{0}$, are as defined in (94). Moreover, there exists a constant $C>0$ independent of $\mathbf{f}_{n}$ such that

$$
\begin{aligned}
& \left(\frac{n \pi}{l}\right)^{\lambda_{k}}\left|\gamma_{n k}\right| \leq C\left\|\widetilde{\mathbf{f}}_{n}^{*}\right\|_{\left(L_{2}(\widetilde{\mathbb{K}})\right)^{2}}, \\
& \left(\frac{n \pi}{l}\right)^{1-\lambda_{1}}\left|\gamma_{n}\right| \leq C\left\|f_{3 n}^{*}\right\|_{L_{2}(\widetilde{\mathbb{K}})}, \\
& \left\|\widetilde{\mathbf{w}}_{n}\right\|_{\left(H^{2}(\widetilde{\mathbb{K}})\right)^{2}}+\frac{n \pi}{l}\left\|\widetilde{\mathbf{w}}_{n}\right\|_{\left(H^{1}(\widetilde{\mathbb{K}})\right)^{2}} \\
& +\left(\frac{n \pi}{l}\right)^{2}\left\|\widetilde{\mathbf{w}}_{n}\right\|_{\left(L_{2}(\widetilde{\mathbb{K}})\right)^{2}} \leq C\left\|\widetilde{\mathbf{f}}_{n}^{*}\right\|_{\left(L_{2}(\widetilde{\mathbb{K}})\right)^{2}}, \\
& \left\|w_{3 n}\right\|_{H^{2}(\widetilde{\mathbb{K}})}+\frac{n \pi}{l}\left\|w_{3 n}\right\|_{H^{1}(\widetilde{\mathbb{K}})} \\
& +\left(\frac{n \pi}{l}\right)^{2}\left\|w_{3 n}\right\|_{L_{2}(\widetilde{\mathbb{K}})} \leq C\left\|f_{3 n}^{*}\right\|_{L_{2}(\widetilde{\mathbb{K}})} .
\end{aligned}
$$

Proof. Relations (95), (96), (97a), (97b), (97c), and (97d) are obtained by a straightforward application of Theorems 10 and 11 to the boundary value problems (93) with $\xi=n \pi / l$ and taking note of the modified right hand side defined in (94).

Theorem 23. Let $\Omega=\widetilde{\mathbb{K}} \times(0, l)$ be a three-dimensional wedge and $\lambda_{k}=k \pi / \omega, k \in \mathbb{N}, \omega \neq \pi$. For each $\mathbf{f}=\left(f_{1}, f_{2}\right.$, $\left.f_{3}\right) \in\left(L_{2}(\Omega)\right)^{3}$, let $\mathbf{u}=\left(u_{1}, u_{2}, u_{3}\right) \in \mathscr{H}_{0}($ curl, div, $\Omega)$ be the solution of the boundary value problem (83). If $\lambda_{k} \neq 2$, $k \in \mathbb{N}$, then there exist unique functions $\Psi_{k} \in H^{\lambda_{k}}(0, l)$ and $\Psi_{3} \in H^{1-\lambda}(0, l)$ such that the solution $\mathbf{u} \in \mathscr{H}_{0}(\operatorname{curl}, \operatorname{div}, \Omega)$ can be split in the form

$$
\begin{aligned}
& \mathbf{u}=\left(w_{1}, w_{2}, w_{3}\right)+\left(s_{1}, s_{2}, s_{3}\right) \\
& \text { with } \mathbf{w}=\left(w_{1}, w_{2}, w_{3}\right) \in\left(H^{2}(\Omega)\right)^{3}, \\
& w_{1}\left(x_{1}, x_{2}, x_{3}\right):=\sum_{n=1}^{\infty} w_{1 n}\left(x_{1}, x_{2}\right) \sin \frac{n \pi x_{3}}{l}, \\
& w_{2}\left(x_{1}, x_{2}, x_{3}\right):=\sum_{n=1}^{\infty} w_{2 n}\left(x_{1}, x_{2}\right) \sin \frac{n \pi x_{3}}{l}, \\
& w_{3}\left(x_{1}, x_{2}, x_{3}\right):=\frac{1}{2} w_{30}\left(x_{1}, x_{2}\right)+\sum_{n=1}^{\infty} u_{3 n}\left(x_{1}, x_{2}\right) \\
& \cdot \cos \frac{n \pi x_{3}}{l}, \quad \\
& s_{1}\left(x_{1}, x_{2}, x_{3}\right):=\sum_{0<\lambda_{k}<2}\left(\Psi_{k}\left(x_{3}\right) * T_{1}\left(r, x_{3}\right)\right) r^{\lambda_{k}-1} \\
& \cdot \sin \left(\left(\lambda_{k}-1\right) \theta\right),
\end{aligned}
$$

$$
\begin{gathered}
s_{2}\left(x_{1}, x_{2}, x_{3}\right):=\sum_{\substack{0<\lambda_{k}<2 \\
\lambda_{k} \neq 1}}\left(\Psi_{k}\left(x_{3}\right) * T_{1}\left(r, x_{3}\right)\right) r^{\lambda_{k}-1} \\
\cdot \cos \left(\left(\lambda_{k}-1\right) \theta\right), \\
s_{3}\left(x_{1}, x_{2}, x_{3}\right):=\left(\Psi_{3}\left(x_{3}\right) * T_{3}\left(r, x_{3}\right)\right) r^{\lambda_{1}} \sin \lambda_{1} \theta \\
\text { if } 0<\lambda_{1}<1,
\end{gathered}
$$

where

$$
\begin{aligned}
\Psi_{k}\left(x_{3}\right) & :=\sum_{n=1}^{\infty} \gamma_{n k} \sin \frac{n \pi x_{3}}{l}, \\
\Psi_{3}\left(x_{3}\right) & :=\frac{1}{2} \gamma_{0}+\sum_{n=1}^{\infty} \gamma_{n} \cos \frac{n \pi x_{3}}{l}, \\
T_{1}\left(r, x_{3}\right) & :=\sum_{n=1}^{\infty} \mathrm{e}^{-(n \pi / l) r} \sin \frac{n \pi x_{3}}{l}, \\
T_{3}\left(r, x_{3}\right) & :=\frac{1}{2}+\sum_{n=1}^{\infty} \mathrm{e}^{-(n \pi / l) r} \cos \frac{n \pi x_{3}}{l} .
\end{aligned}
$$

In (98), the symbol “*” denotes the convolution product in the variable $x_{3}$; that is,

$$
\begin{aligned}
& \Psi_{k}\left(x_{3}\right) * T_{1}\left(r, x_{3}\right):=\sum_{n=1}^{\infty} \gamma_{n k} \mathrm{e}^{-(n \pi / l) r} \sin \frac{n \pi x_{3}}{l}, \\
& \Psi_{3}\left(x_{3}\right) * T_{3}\left(r, x_{3}\right):=\frac{1}{2} \gamma_{0}+\sum_{n=1}^{\infty} \gamma_{n} \mathrm{e}^{-(n \pi / l) r} \cos \frac{n \pi x_{3}}{l} .
\end{aligned}
$$

The coefficients $\gamma_{n k}$ and $\gamma_{n}$ are as defined in Theorem 22.

Proof. The expressions (98), (99), and (100) are direct consequences of Lemma 21 and Theorem 22, taking into consideration relations (35) and (36). The inequalities (97c) and (97d) and Parseval's identity (86) imply that the Fourier coefficients $\mathbf{w}_{n}=\left(w_{1 n}, w_{2 n}, w_{3 n}\right), n \in \mathbb{N}_{0}$, satisfy the estimate

$$
\begin{aligned}
& \sum_{n=1}^{\infty}\left\{\left\|\mathbf{w}_{n}\right\|_{\left(H^{2}(\widetilde{\mathbb{K}})\right)^{3}}^{2}+\left(\frac{n \pi}{l}\right)^{2}\left\|\mathbf{w}_{n}\right\|_{\left(H^{1}(\widetilde{\mathbb{K}})\right)^{3}}^{2}\right. \\
& \left.+\left(\frac{n \pi}{l}\right)^{4}\left\|\mathbf{w}_{n}\right\|_{\left(L_{2}(\widetilde{\mathbb{K}})\right)^{3}}^{2}\right\} \leq C\left\|\mathbf{f}^{*}\right\|_{\left(L_{2}(\Omega)\right)^{3}}^{2}<\infty
\end{aligned}
$$

Hence, $\mathbf{w} \in\left(H^{2}(\Omega)\right)^{3}$; see [24, Theorem 3.2]. The inequalities (97a) and (97b) lead to the estimates

$$
\begin{aligned}
& \sum_{n=1}^{\infty}\left(\frac{n \pi}{l}\right)^{2 \lambda_{k}}\left|\gamma_{n k}\right|^{2} \leq C \sum_{n=0}^{\infty}\left\|\widetilde{\mathbf{f}}_{n}^{*}\right\|_{\left(L_{2}(\widetilde{\mathbb{K}})\right)^{2}}^{2} \\
& \quad \leq C\left\|\widetilde{\mathbf{f}}^{*}\right\|_{\left(L_{2}(\Omega)\right)^{2}}^{2}<\infty,
\end{aligned}
$$




$$
\begin{aligned}
& \sum_{n=0}^{\infty}\left(1+\left(\frac{n \pi}{l}\right)^{2\left(1-\lambda_{1}\right)}\right)\left|\gamma_{3 n}\right|^{2} \leq C \sum_{n=0}^{\infty}\left\|f_{3 n}^{*}\right\|_{L_{2}(\widetilde{\mathbb{K}})}^{2} \\
& \quad \leq C\left\|f_{3}^{*}\right\|_{L_{2}(\Omega)}^{2}
\end{aligned}
$$

which by the generalized Riesz-Fischer theorem imply the existence of functions $\Psi_{k} \in H^{\lambda_{k}}(0, l)$ and $\Psi_{3} \in H^{1-\lambda_{1}}(0, l)$ whose Fourier coefficients are $\left\{\gamma_{n k}: n \in \mathbb{N}\right\}$ and $\left\{\gamma_{n}: n \in \mathbb{N}_{0}\right\}$, respectively.

5.2. Singularities near Polyhedral Edges. In this subsection, we consider and analyze the regularity properties of the solution of the Maxwell equations (25) in general threedimensional domains with straight edges bounded by plain faces, that is, polyhedral edges. In fact it would be sufficient for us to consider three-dimensional domains of the form $Q=\Omega \times(0, l)$, where $l>0$ is a real constant and $\Omega \subset \mathbb{R}^{2}$ is a general bounded domain with piecewise smooth boundary $\Gamma$. We will use the same notations as in Section 3 for $\Omega$ and $Q$.

Thus the edges of $Q$ are $E_{j}=A_{j} \times(0, l), j=1, \ldots, J$, and the boundary $\partial Q$ is the union of the disjoint faces $T_{j}:=$ $\Gamma_{j} \times(0, l), j=1, \ldots, J$, and the two bases $T_{j+1}:=\omega \times\{0\}$ and $T_{J+2}:=\Omega \times\{l\}$. We assume that, for $\left(x_{1}, x_{2}, x_{3}\right) \in Q$, $\left(x_{1}, x_{2}\right) \in \Omega$ and $x_{3} \in(0, l)$. We associate with each edge $E_{j}$ a three-dimensional wedge $G_{j}:=\widetilde{\mathbb{K}}_{j} \times(0, l)$, where $\widetilde{\mathbb{K}}_{j} \subset$ $\Omega$ is a circular sector; see (17) and (18). Thus the boundary $\partial G_{j}$ of $G_{j}$ is the union of the disjoint faces $T_{j 0}:=\Gamma_{j 0} \times(0, l)$, $T_{j 1}:=\Gamma_{j} \times(0, l), T_{j 2}:=\Gamma_{j+1} \times(0, l), T_{j 3}:=\widetilde{\mathbb{K}}_{j} \times\{0\}$, and $T_{j 4}:=\widetilde{\mathbb{K}}_{j} \times\{l\}$. Furthermore, we define on each $G_{j}$ a smooth truncation function $\eta_{j}=\eta_{j}\left(x_{1}, x_{2}\right)=\eta_{j}\left(r_{j}\right)$; see (19).

For $\mathbf{f} \in\left(L_{2}(Q)\right)^{3}$ and $\alpha \neq 0$, let $\mathbf{u} \in \mathscr{H}_{0}(\operatorname{curl}, \operatorname{div}, Q)$ be the variational solution of the Maxwell equations (25). Then the function $\mathbf{u}_{j}:=\eta_{j} \mathbf{u}$ which is defined on the wedge $G_{j}$ belongs to the space $\mathscr{H}_{0}$ (curl, div, $G_{j}$ ) and is the unique weak solution of the boundary value problem

$$
\begin{aligned}
& \operatorname{curlcurl} \mathbf{u}_{j}-\operatorname{grad} \operatorname{div} \mathbf{u}_{j}=\mathbf{f}_{j} \quad \text { in } G_{j}, \\
& \qquad \begin{aligned}
& \mathbf{u}_{j} \wedge \mathbf{n}= \mathbf{0} \\
& \quad \text { on } T_{j 1} \cup T_{j 2} \cup T_{j 3} \cup T_{j 4}, \\
& \operatorname{div} \mathbf{u}_{j}= 0 \\
& \quad \text { on } T_{j 1} \cup T_{j 2} \cup T_{j 3} \cup T_{j 4}, \\
& \mathbf{u}_{j}=\mathbf{0} \text { on } T_{j 0},
\end{aligned}
\end{aligned}
$$

where the function $\mathbf{f}_{j}=\left(f_{1 j}, f_{2 j}, f_{3 j}\right) \in\left(L_{2}\left(G_{j}\right)\right)^{3}$ is given by

$$
\begin{aligned}
f_{1 j}\left(x_{j}, y_{j}, x_{3}\right):= & \eta_{j}\left(f_{1}+\alpha^{2} u_{1}\right)-u_{1} \Delta_{x_{1} x_{2}} \eta_{j} \\
& -2 \nabla_{x_{1} x_{2}} \eta_{j} \cdot \nabla_{x_{1} x_{2}} u_{1},
\end{aligned}
$$

$$
\begin{aligned}
f_{2 j}\left(x_{j}, y_{j}, x_{3}\right):= & \eta_{j}\left(f_{2}+\alpha^{2} u_{2}\right)-u_{2} \Delta \eta_{j}-2 \nabla_{x_{1} x_{2}} \eta_{j} \\
& \cdot \nabla_{x_{1} x_{2}} u_{2}, \\
f_{3 j}\left(x_{j}, y_{j}, x_{3}\right):= & \eta_{j}\left(f_{3}+\alpha^{2} u_{3}\right)-u_{3} \Delta \eta_{j}-2 \nabla_{x_{1} x_{2}} \eta_{j} \\
& \cdot \nabla_{x_{1} x_{2}} u_{3} .
\end{aligned}
$$

We observe that problems (83) and (103) are similar and therefore their regularity properties are described by Theorem 23. On the other hand, since the solution $\mathbf{u} \in$ $\mathscr{H}_{0}$ (curl, div, Q) of problem (25) coincides with the solution $\mathbf{u}_{j} \in \mathscr{H}_{0}$ (curl, div, $G_{j}$ ) of problem (103) near the edge $E_{j}$ of $Q$, Theorem 23 also addresses the regularity properties of the solution $\mathbf{u}$ of (25). Thus we obtain the following theorem.

Theorem 24. For $f \in L_{2}(Q)^{3}$ and $\alpha \neq 0$, let $\mathbf{u} \in \mathscr{H}_{0}$ (curl, $\operatorname{div}, Q)$ be the variational solution of the boundary value problem (25). Let $\lambda_{j k}:=k \pi / \omega_{j}, k \in \mathbb{N}$, and $\omega_{j} \neq \pi, j=1, \ldots$, J. If $\lambda_{j k} \neq 2, k \in \mathbb{N}, j=1, \ldots, J$, then there exist functions $\Psi_{j k} \in H^{\lambda_{j k}}(0, l)$ and $\Psi_{j} \in H^{1-\lambda_{j 1}}(0, l)$ such that the solution $u \in \mathscr{H}_{0}($ curl, div, Q) can be represented in the form

$$
\mathbf{u}=\left(w_{1}, w_{2}, w_{3}\right)+\left(s_{1}, s_{2}, s_{3}\right)
$$

with $\mathbf{w} \in\left(H^{2}(Q)\right)^{3}$

$$
\begin{aligned}
& s_{1}\left(x_{1}, x_{2}, x_{3}\right):=\sum_{\substack { j=1 \\
\begin{subarray}{c}{0<\lambda_{j k}<2 \\
\lambda_{j k} \neq 1{ j = 1 \\
\begin{subarray} { c } { 0 < \lambda _ { j k } < 2 \\
\lambda _ { j k } \neq 1 } }\end{subarray}}^{N}\left(\Psi_{j k}\left(x_{3}\right) * T_{j}\left(r_{j}, x_{3}\right)\right) \\
& \cdot r_{j}^{\lambda_{j k}-1} \sin \left(\left(\lambda_{j k}-1\right) \theta_{j}\right), \\
& s_{2}\left(x_{1}, x_{2}, x_{3}\right):=\sum_{j=1}^{N} \sum_{\substack{0<\lambda_{k}<2 \\
\lambda_{j k} \neq 1}}\left(\Psi_{j k}\left(x_{3}\right) * T_{j}\left(r_{j}, x_{3}\right)\right) \\
& \cdot r_{j}^{\lambda_{j k}-1} \cos \left(\left(\lambda_{j k}-1\right) \theta_{j}\right), \\
& s_{3}\left(x_{1}, x_{2}, x_{3}\right):=\sum_{j=1}^{N}\left(\Psi_{j}\left(x_{3}\right) * T_{3 j}\left(r_{j}, x_{3}\right)\right) r_{j}^{\lambda_{j 1}} \\
& \cdot \sin \lambda_{j 1} \theta_{j} \quad \text { if } 0<\lambda_{j 1}<1 .
\end{aligned}
$$

The functions $T_{j}$ and $T_{3 j}$ are fixed kernels given by

$$
\begin{gathered}
T_{j}\left(r_{j}, x_{3}\right):=\sum_{n=1}^{\infty} \mathrm{e}^{-(n \pi / l) r_{j}} \sin \frac{n \pi x_{3}}{l}, \\
T_{3 j}\left(r_{j}, x_{3}\right):=\sum_{n=1}^{\infty} \mathrm{e}^{-(n \pi / l) r_{j}} \cos \frac{n \pi x_{3}}{l} .
\end{gathered}
$$


The coefficients $\Psi_{j k}$ and $\Psi_{j}$ are defined explicitly by

$$
\begin{aligned}
\Psi_{j k}\left(x_{3}\right) & :=\sum_{n=1}^{\infty} \gamma_{j n k} \sin \frac{n \pi x_{3}}{l}, \\
\Psi_{j}\left(x_{3}\right) & :=\frac{1}{2} \gamma_{j 0}+\sum_{n=1}^{\infty} \gamma_{j n} \cos \frac{n \pi x_{3}}{l},
\end{aligned}
$$

where the coefficients $\gamma_{j n k}$ and $\gamma_{j n}$ are as defined in (30).

\section{Conflict of Interests}

The author declares that there is no conflict of interests regarding the publication of this paper.

\section{Acknowledgments}

This work was supported by the Alexander von Humboldt Foundation $(\mathrm{AvH})$, Bonn, Germany, and the Abdus Salam International Centre for Theoretical Physics (ICTP), Trieste, Italy.

\section{References}

[1] M. Dauge, Elliptic Boundary Value Problems on Corner Domains, vol. 1341 of Lecture Notes in Mathematics, Springer, Berlin, Germany, 1988.

[2] P. Grisvard, Singularities in Boundary Value Problems, Masson, Paris, France, 1992.

[3] P. Grisvard, Elliptic Problems in Nonsmooth Domains, Pitman Advanced Publishing Program, Boston, Mass, USA, 1985.

[4] M. Sh. Birman and M. Z. Solomyak, " $L_{2}$-theory of the Maxwell operator in arbitrary domains," Russian Mathematical Surveys, vol. 42, no. 6, pp. 75-96, 1987.

[5] A.-S. Bonnet-Ben Dhia, C. Hazard, and S. Lohrengel, "A singular field method for the solution of Maxwell's equations in polyhedral domains," SIAM Journal on Applied Mathematics, vol. 59, no. 6, pp. 2028-2044, 1999.

[6] M. Moussaoui, "Espaces $\mathrm{H}(\operatorname{div}, \operatorname{rot}, \Omega$ ) dans un polygon plan," Comptes Rendus de l'Académie des Sciences de Paris, Série I, vol. 322, pp. 225-229, 1996.

[7] C. Hazard and S. Lohrengel, "A singular field method for Maxwell's equations: numerical aspects for 2D magnetostatics," SIAM Journal on Numerical Analysis, vol. 40, no. 3, pp. 10211040, 2002.

[8] C. Hazard and M. Lenoir, "On the solution of time-harmonic scattering problems for Maxwell's equations," SIAM Journal on Mathematical Analysis, vol. 27, no. 6, pp. 1597-1630, 1996.

[9] S. Lohrengel, Etude mathématique et résolution numérique des équations de Maxwell dans un domaine non régulier [Ph.D. thesis], University of Paris, Paris, France, 1998.

[10] M. Costabel, "A remark on the regularity of solutions of Maxwell's equations on Lipschitz domains," Mathematical Methods in the Applied Sciences, vol. 12, no. 4, pp. 365-368, 1990.

[11] B. Nkemzi, "Optimal convergence recovery for the Fourierfinite-element approximation of Maxwell's equations in nonsmooth axisymmetric domains," Applied Numerical Mathematics, vol. 57, no. 9, pp. 989-1007, 2007.
[12] B. Nkemzi, "On singularities of solution of Maxwell's equations in axisymmetric domains with conical points," Mathematical Methods in the Applied Sciences, vol. 30, no. 8, pp. 877-888, 2007.

[13] B. Nkemzi, "On the solution of Maxwell's equations in polygonal domains," Mathematical Methods in the Applied Sciences, vol. 29, no. 9, pp. 1053-1080, 2006.

[14] B. Nkemzi, "On the solution of Maxwell's equations in axisymmetric domains with edges," Zeitschrift für Angewandte Mathematik und Mechanik, vol. 85, pp. 570-592, 2005.

[15] M. Costabel, M. Dauge, and S. Nicaise, "Singularities of Maxwell interface problems," Mathematical Modelling and Numerical Analysis, vol. 33, no. 3, pp. 627-649, 1999.

[16] M. Costabel and M. Dauge, "Singularities of electromagnetic fields in polyhedral domains," Archive for Rational Mechanics and Analysis, vol. 151, no. 3, pp. 221-276, 2000.

[17] V. A. Kondratev, "Singularities of solutions of the Derichlet problem for an elliptic equation of second order in the neighbourhood of an edge," Differentsial'nye Uravneniya, vol. 13, pp. 2026-2032, 1967 (Russian), English Translation in: Differential Equations, vol. 13, pp. 1411-1415, 1967.

[18] B. Heinrich, "The Fourier-finite-element method for Poisson's equation in axisymmetric domains with edges," SIAM Journal on Numerical Analysis, vol. 33, no. 5, pp. 1885-1911, 1996.

[19] R. Leis, Initial Boundary Value Problems in Mathematical Physics, John Wiley \& Sons, New York, NY, USA, 1986.

[20] R. Leis, "Exterior boundary value problems in mathematical physics," in Trends in Applications of Pure Mathematics to Mechanics, H. Zorski, Ed., vol. 11, pp. 187-203, Pitman, 1979.

[21] V. Girault and P.-A. Raviart, Finite Element Methods for NavierStokes Equations, Springer, Berlin, Germany, 1986.

[22] A. Alonso and A. Valli, "Unique solvability for high-frequency heterogeneous time-harmonic Maxwell equations via the Fredholm alternative theory," Mathematical Methods in the Applied Sciences, vol. 21, no. 6, pp. 463-477, 1998.

[23] J. E. Santos and D. Sheen, "On the existence and uniqueness of solutions to Maxwell's equations in bounded domains with application to magnetotellurics," Mathematical Models and Methods in Applied Sciences, vol. 10, no. 4, pp. 615-628, 2000.

[24] B. Nkemzi and M. Jung, "Flux intensity functions for the Laplacian at polyhedral edges," International Journal of Fracture, vol. 175, no. 2, pp. 167-185, 2012.

[25] R. V. Churchill, Fourier Series and Boundary Value Problems, McGraw-Hill, New York, NY, USA, 1963.

[26] A. Khinchin, A Course of Mathematical Analysis, Hindustan Publishing Corporation, New Delhi, India, 1960. 


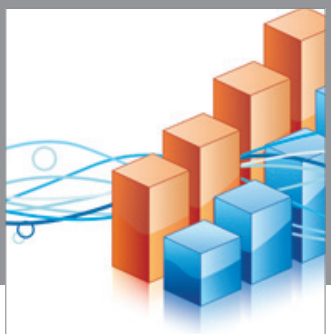

Advances in

Operations Research

vatem alat4

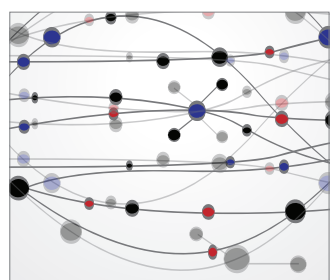

\section{The Scientific} World Journal
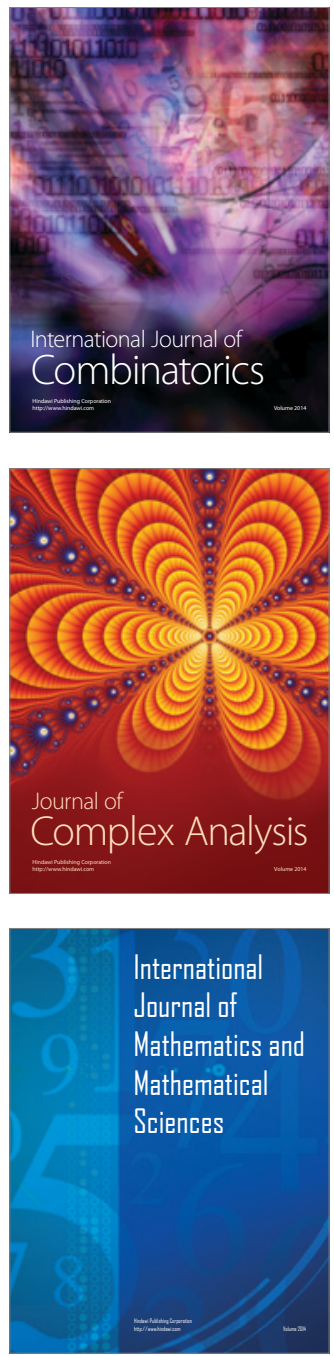
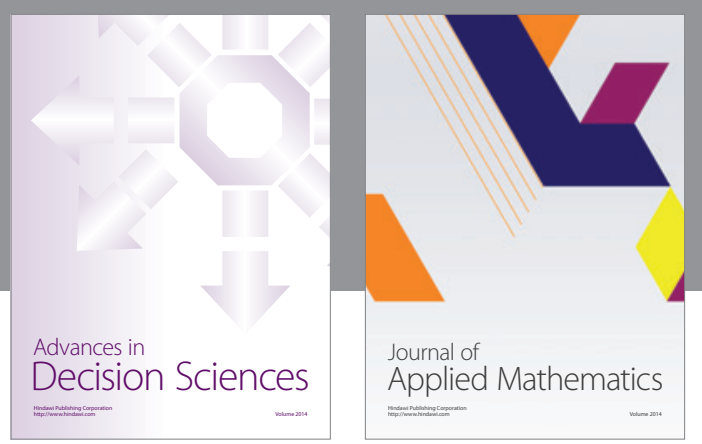

Algebra

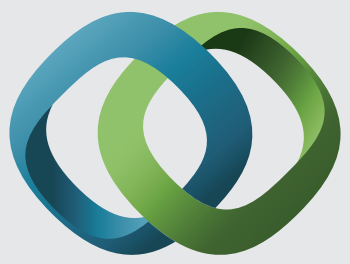

\section{Hindawi}

Submit your manuscripts at

http://www.hindawi.com
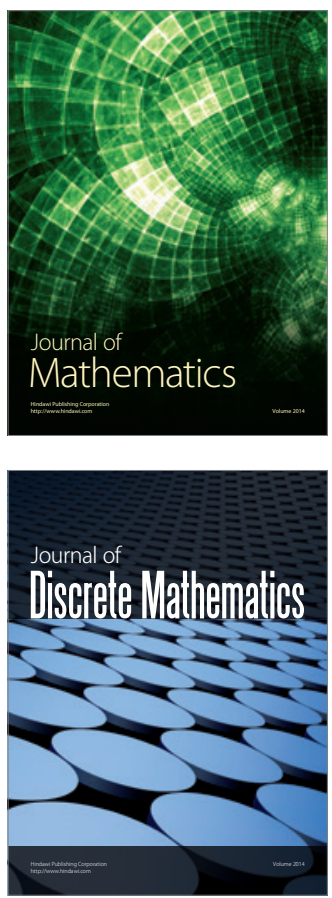

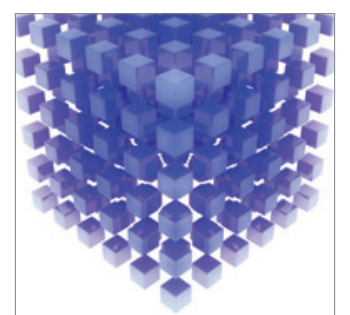

Mathematical Problems in Engineering
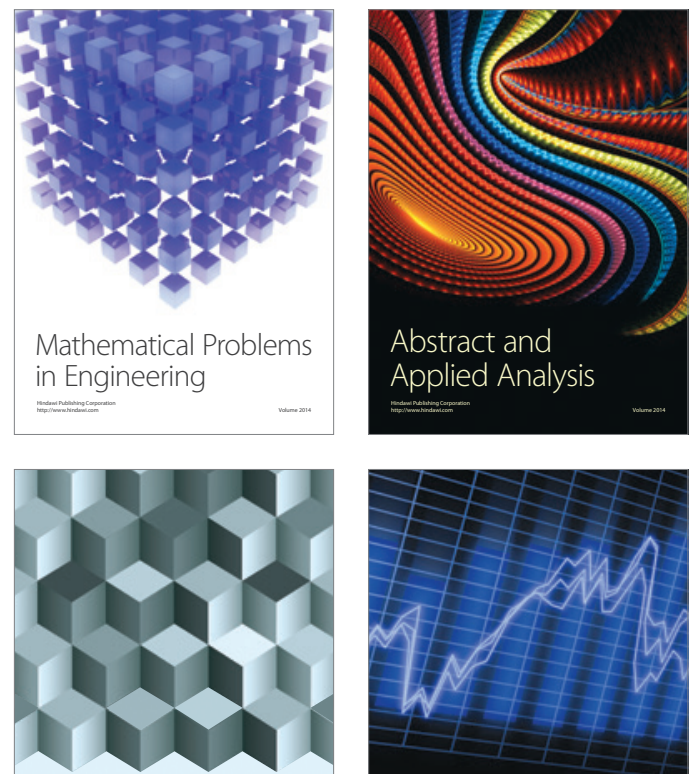

Journal of

Function Spaces

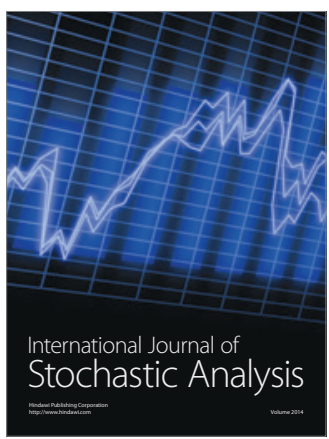

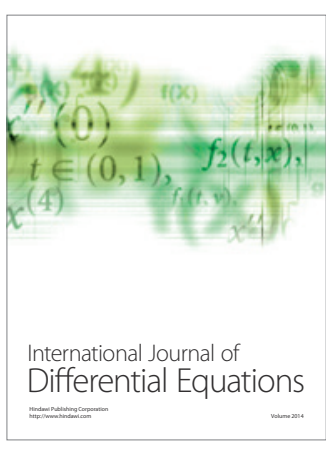
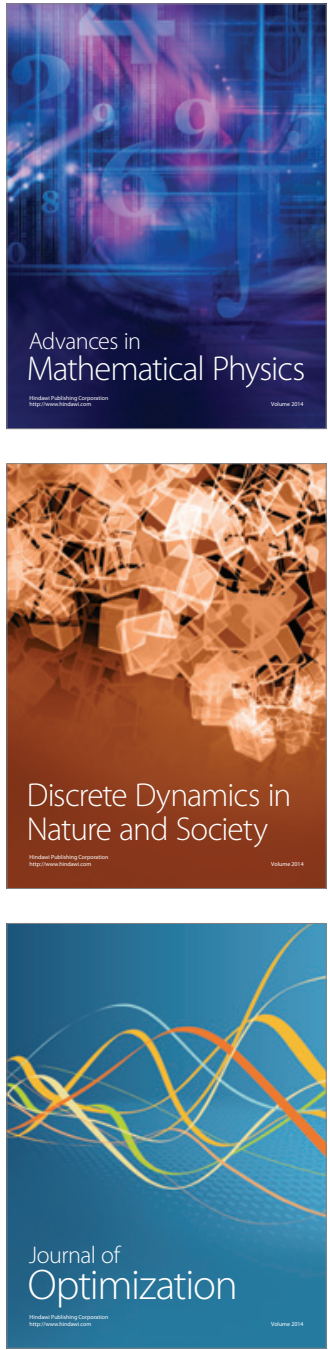